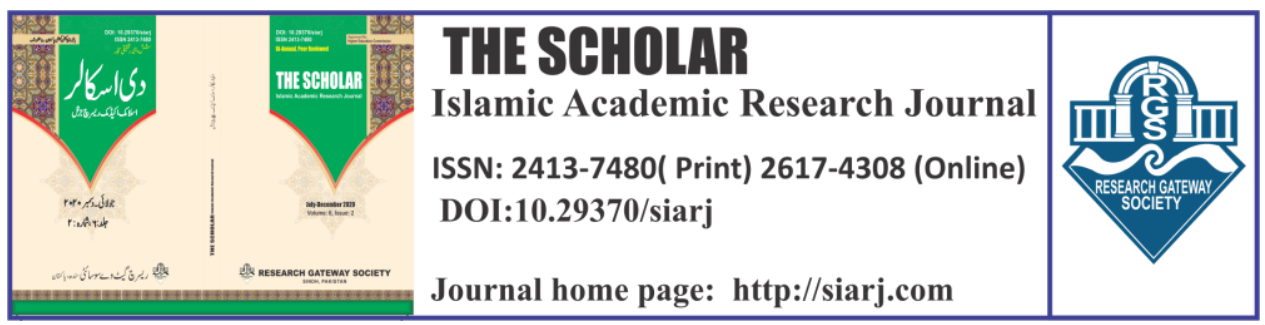

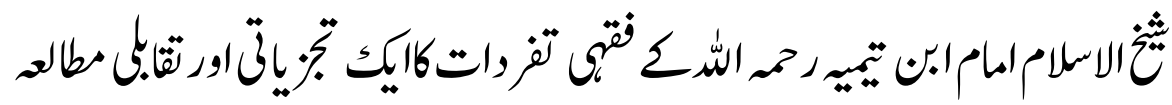

\title{
AN ANALYTICAL AND COMPARATIVE STUDY OF SHAYKH-UL-ISLAM IMAM IBN TAYMIYYAH'S JURISPRUDENTIAL DIFFERENCES
}

\section{Muhammad Fazal Haq}

Ph.D. Scholar, Department of Islamic Studies,

The Islamia University of Bahawalpur

Email: turabi5654@gmail.com

ORCID ID:

https://orcid.org/0000-0002-9880-6437

\section{Hafiz Muhammad Nasrullah}

Assistant Professor, Department of Islamic Studies, The Islamia University of Bahawalpur.

Email: drhafiznasrullah@gmail.com

ORCID ID:

https://orcid.org/0000-0002-1259-0437

To cite this article:

Muhammad Fazal Haq, Hafiz Muhammad Nusrullah. "URDU-AN ANALYTICAL AND COMPARATIVE STUDY OF SHAYKH-UL-ISLAM IMAM IBN TAYMIYYAH'S

JURISPRUDENTIAL DIFFERENCES." The Scholar-Islamic Academic Research Journal 6, No. 2 (December 23, 2020): 20-42.

To link to this article: https://doi.org/10.29370/siarj/issue11urduar2

Journal

Publisher

DOI:

URL:

License:

Journal homepage

Published online:
The Scholar Islamic Academic Research Journal

Vol. 6, No. 2 || July-December 2020 || P. 20-42

Research Gateway Society

10.29370/siarj/issue11urduar2

https://doi.org/10.29370/siarj/ issue1 1urduar2

Copyright c 2017 NC-SA 4.0

www.siarj.com

2020-12-23
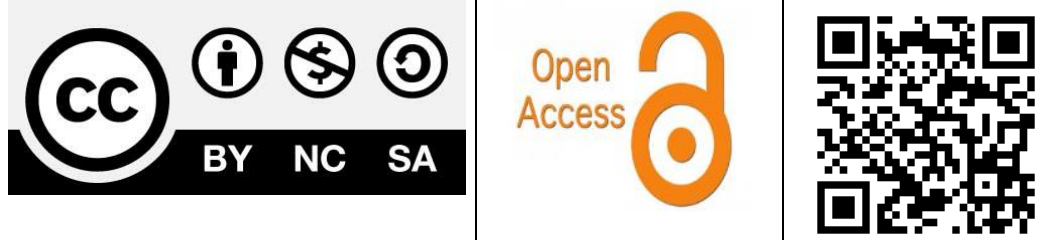


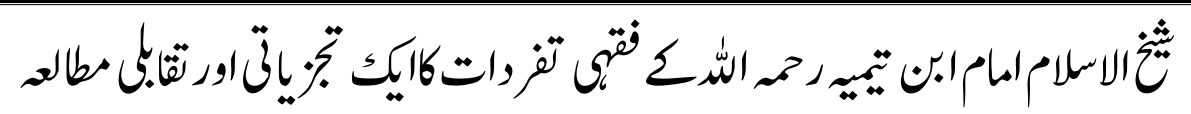 \\ AN ANALYTICAL AND COMPARATIVE STUDY OF SHAYKH- UL-ISLAM IMAM IBN TAYMIYYAH'S JURISPRUDENTIAL DIFFERENCES
}

Muhammad Fazal Haq, Hafiz Muhammad Nasrullah

\begin{abstract}
:
Taqi ad Din Ahamad ibn Taymiyyah remarkable, recognized and medieval Sunni Theologian, jurisconsult, logician and great reformer today, he is known by the title of Sheikh-ul-Islam. He was the member of Hanbali School of jurisprudence and well known personality and figure in his own life period. He had great and matchless command and mastery overall knowledge of that time. He led the Muslims community of his age in every field of life. This made him unpopular with majority scholar of the time. Therefore, he was imprisoned several times. See, the miracles of the nature, he died in prison. He adopted a separate way of Islamic jurisprudence in that research . By this he had his own opinions and thoughts about jurisprudence, some of these are different from four schools of jurisprudence. His different opinions and thoughts are called "Fiqhi tafarudat" "فقهى تفرداتّ"Which are on the base of Quran and Sunnah.
\end{abstract}

KEYWORDS: Ibn e Tamiyyah, Fiqhi Tafarudat, Analytical study, Quran, Sunnah

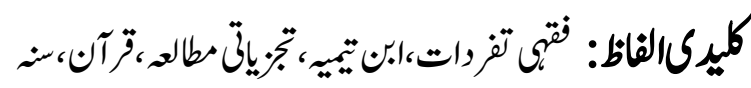

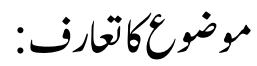




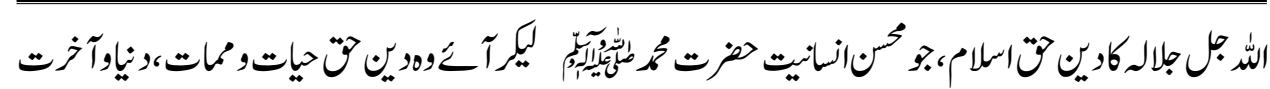

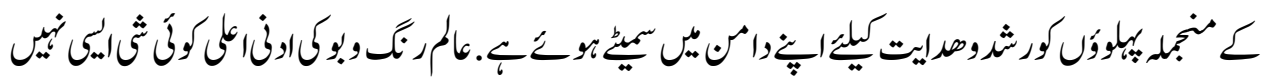

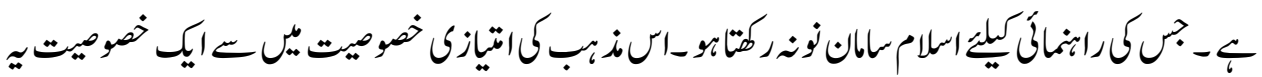

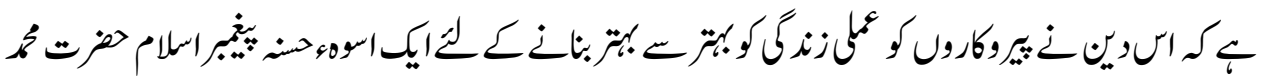

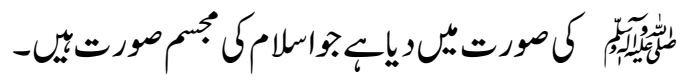

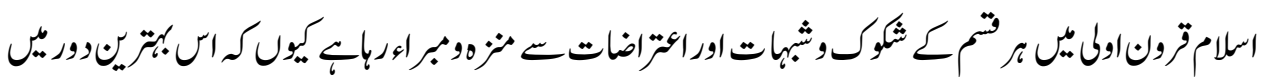

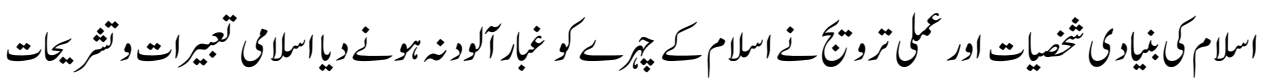

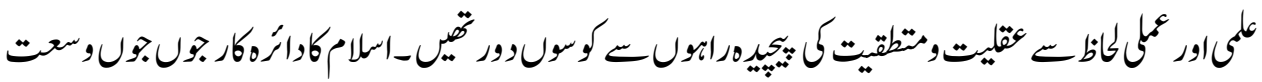

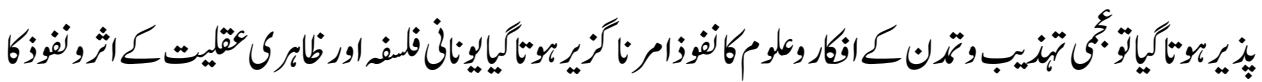

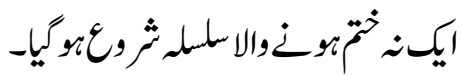

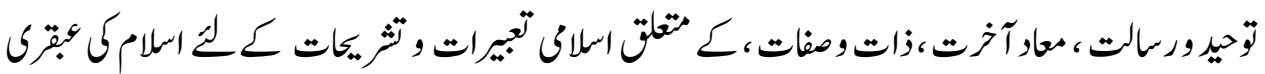

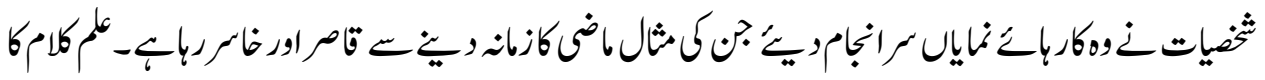

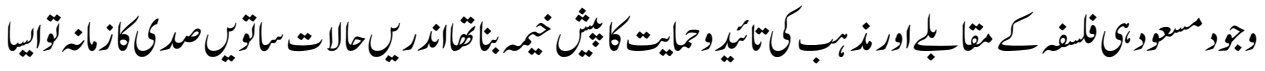

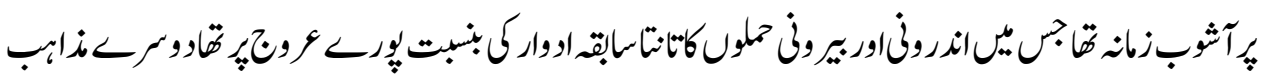

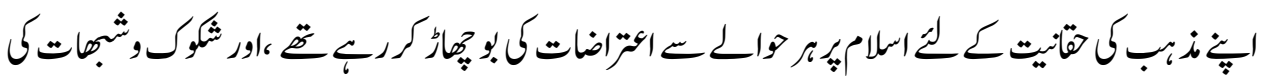

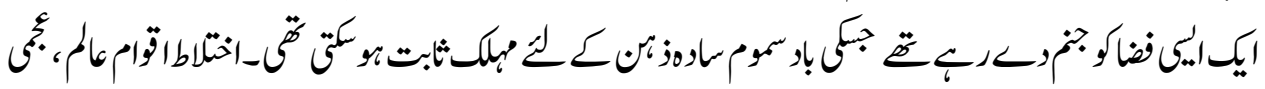

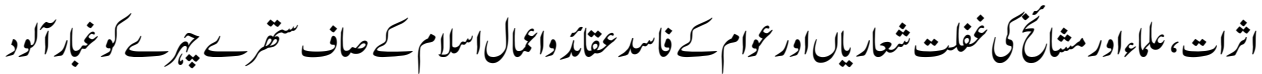

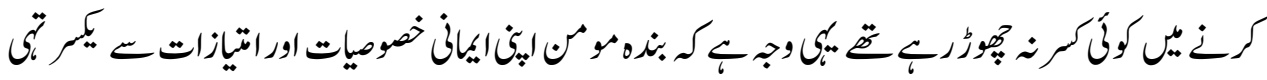

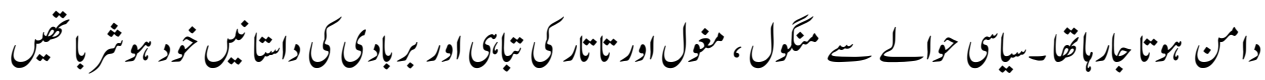

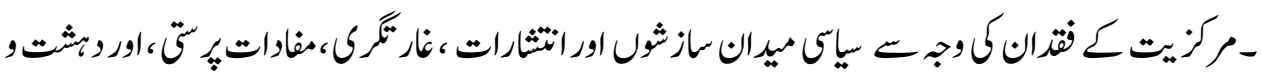

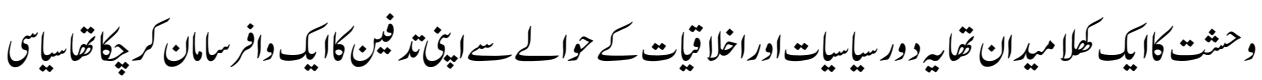

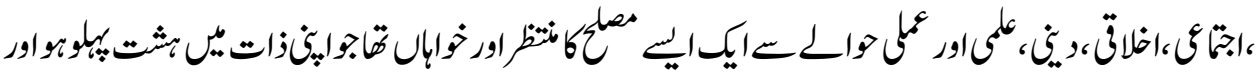

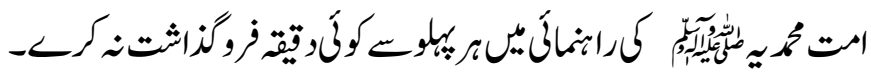

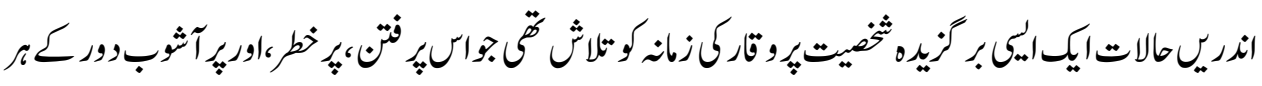




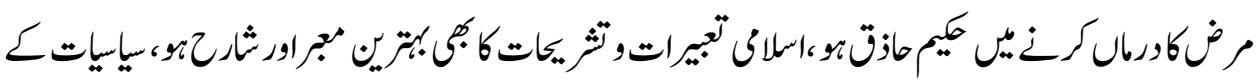

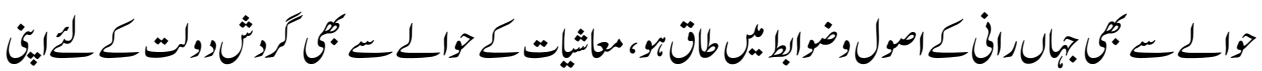

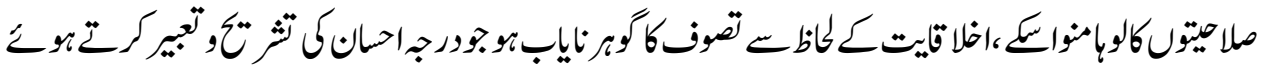

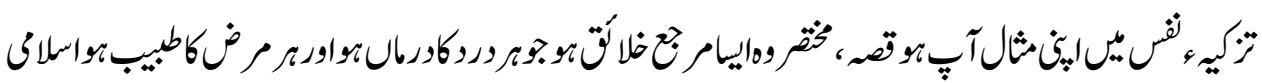

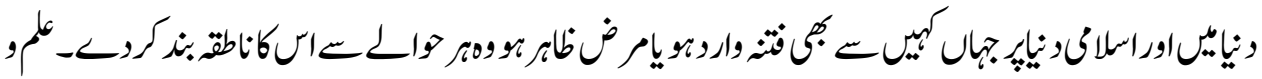

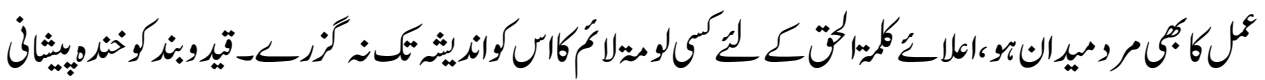

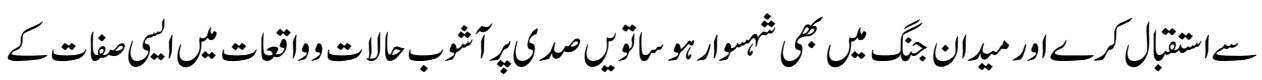

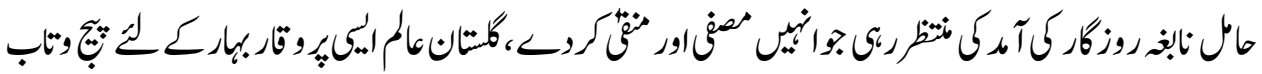

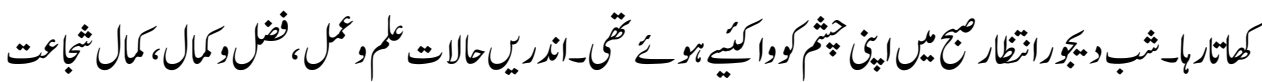

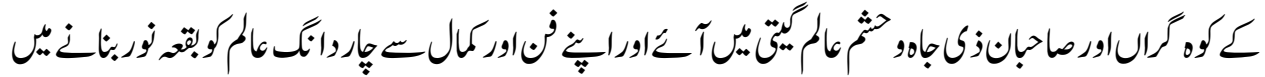

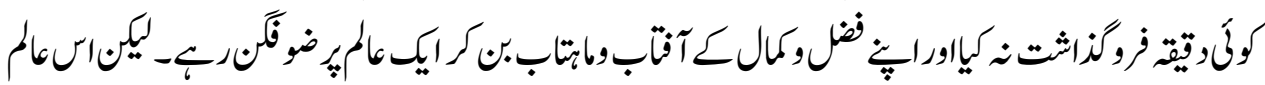

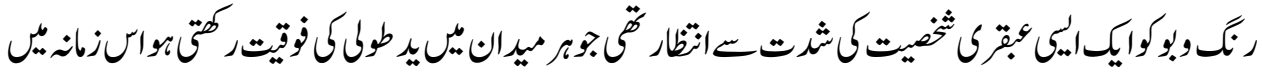

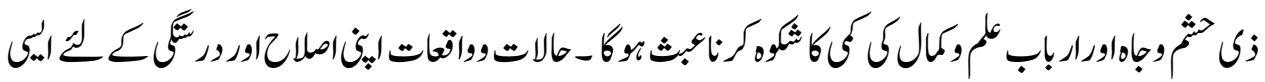

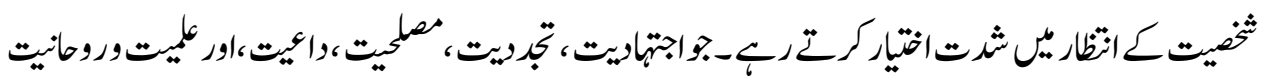

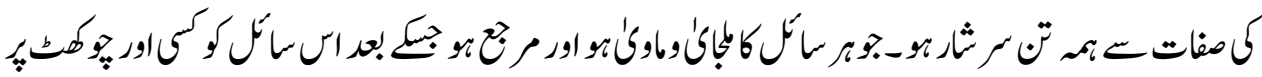

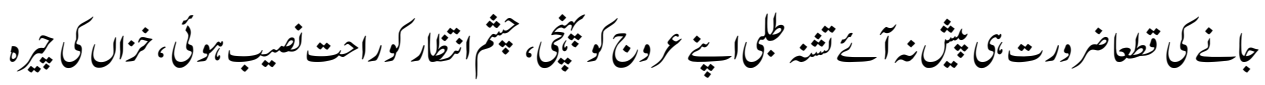

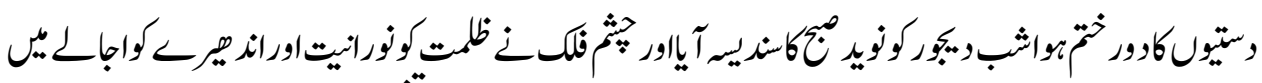

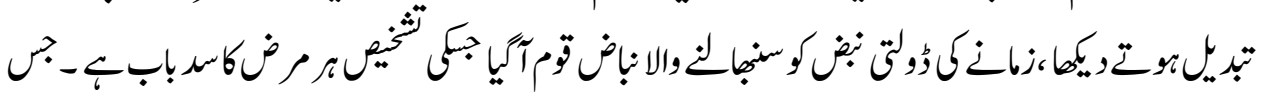

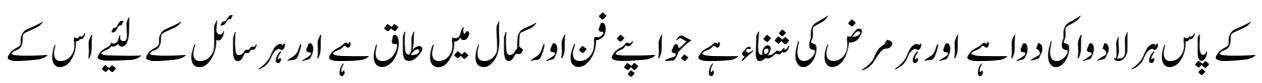

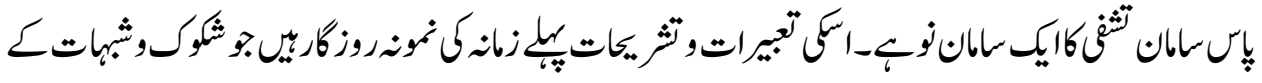

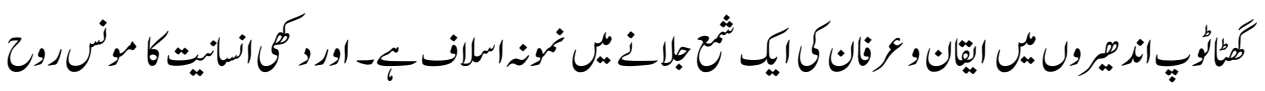

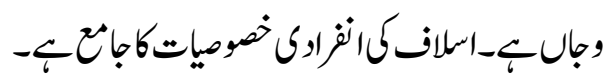

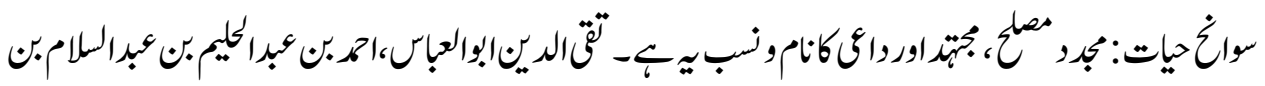

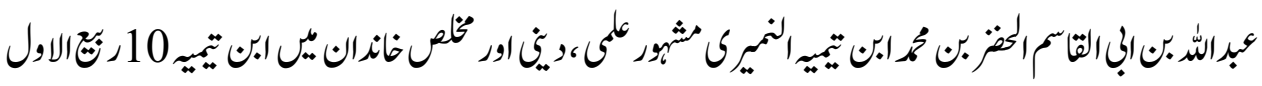




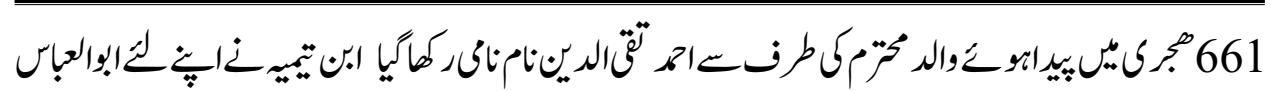

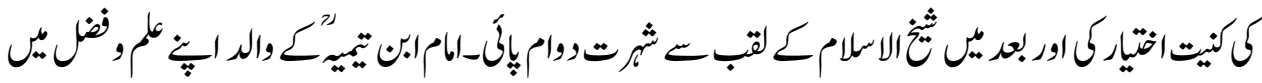

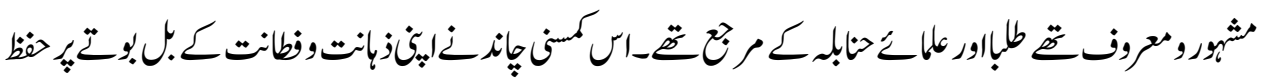

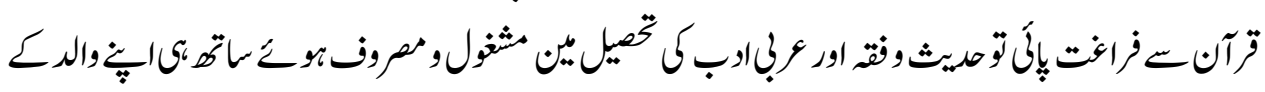

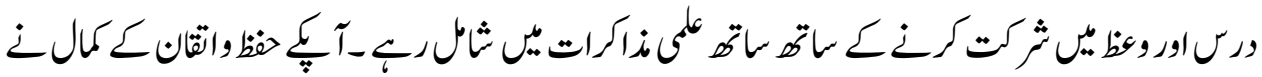

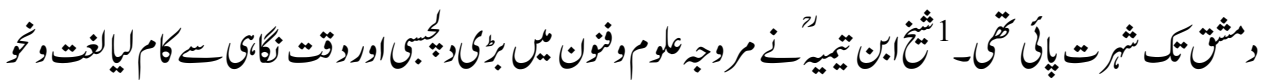

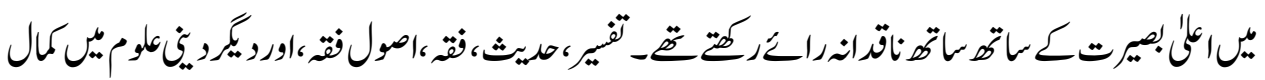

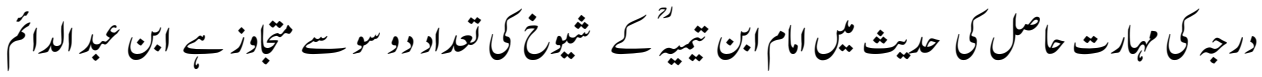

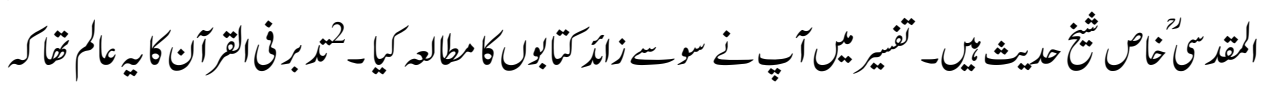

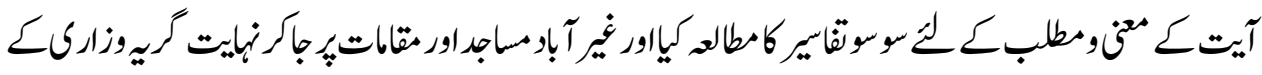

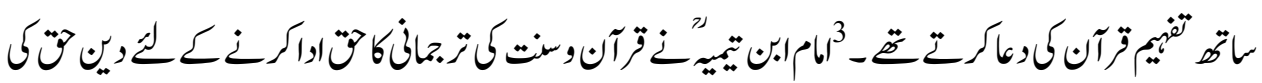

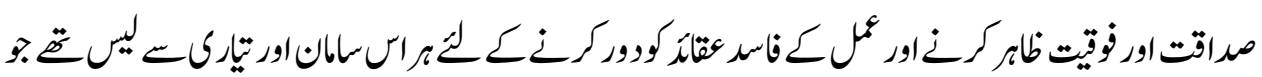

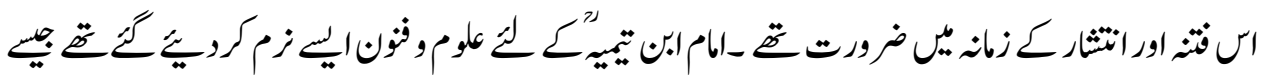

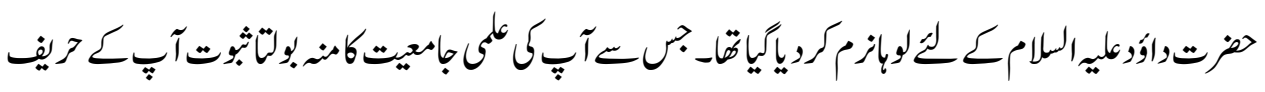

${ }^{1}$ Salehi, Abu Abdullah Muhammad bin Ahmad bin Abdul Hadi Damascus (705-744 AH) Al-Aqood Al-Dariya from Manaqib Shaykh al-Islam Ibn Taymiyyah, Research: Abu Musab Talat bin Fawad Halwa Ni , Cairo, Egypt, I: 1, 1422 AH 2002, p. 7.

${ }^{2}$ Nadwi Syed Abu Al-Hassan Ali, History of Da'wah and Commitment, Majlis-e-Nashriyat-e-Islam, Karachi, Pakistan, p.38..

${ }^{3}$ Salehi, Al-Aqood Al-Dariya, p: 25. 


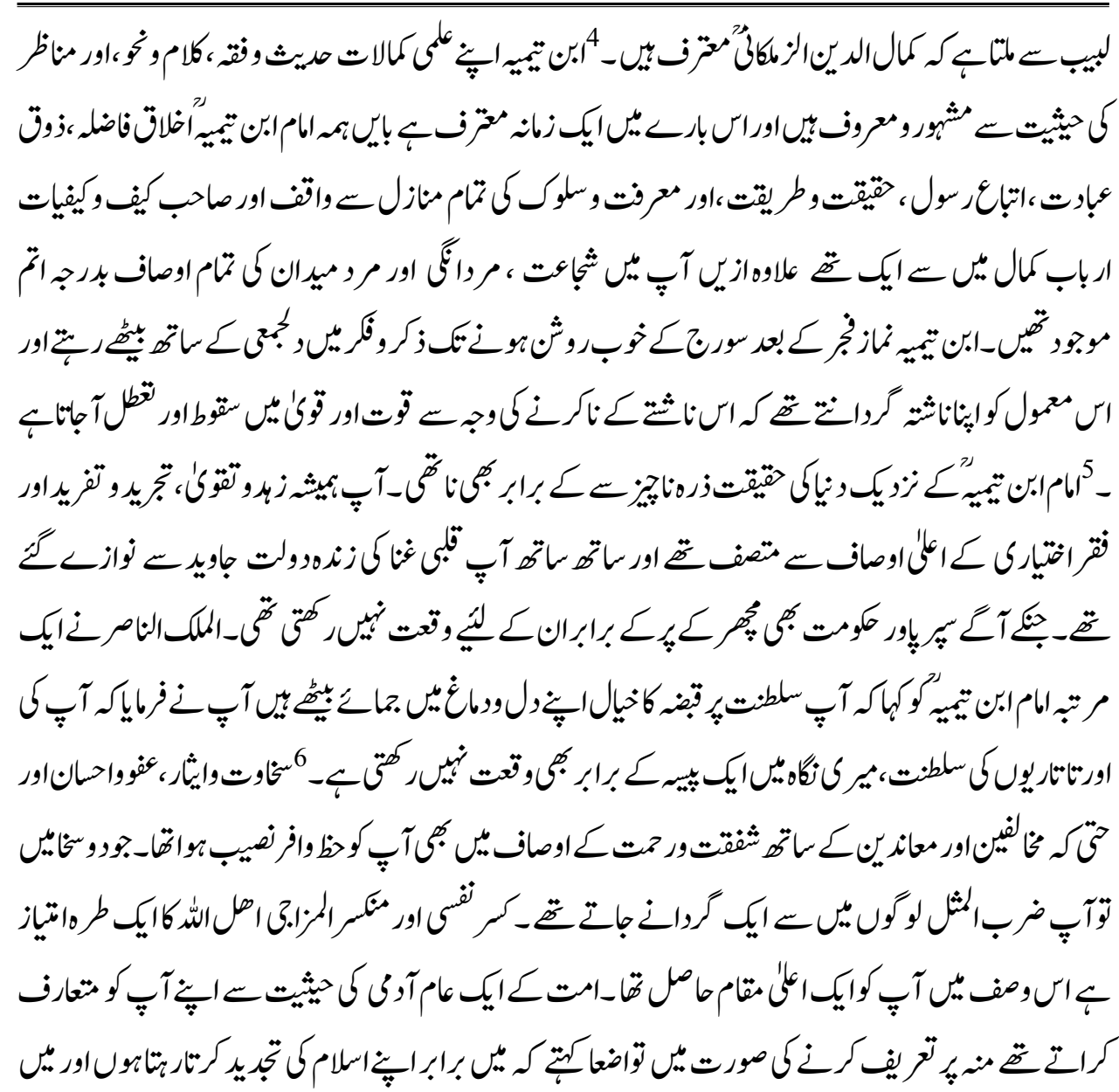

${ }^{4}$ Karami, Mar'i ibn Yusuf Hanbali (1033 AH), Al-Kawakib al-Dariyat fi Manaqib al-Mujtahid Ibn Taymiyyah,p.60.

${ }^{5}$ Ibn Nasser al-Din, Muhammad ibn Abu Bakr Damascus Shafi'i (842 AH) Al-Rad al-Wafir Ali from Za'am: 1980, p. 120.

${ }^{6}$ Karami, Mar'i bin Yusuf Hanbali (1033 AH) ), Al-Kawakib al-Dariyat fi Manaqib al-Mujtahid Ibn Taymiyyah,p.99 


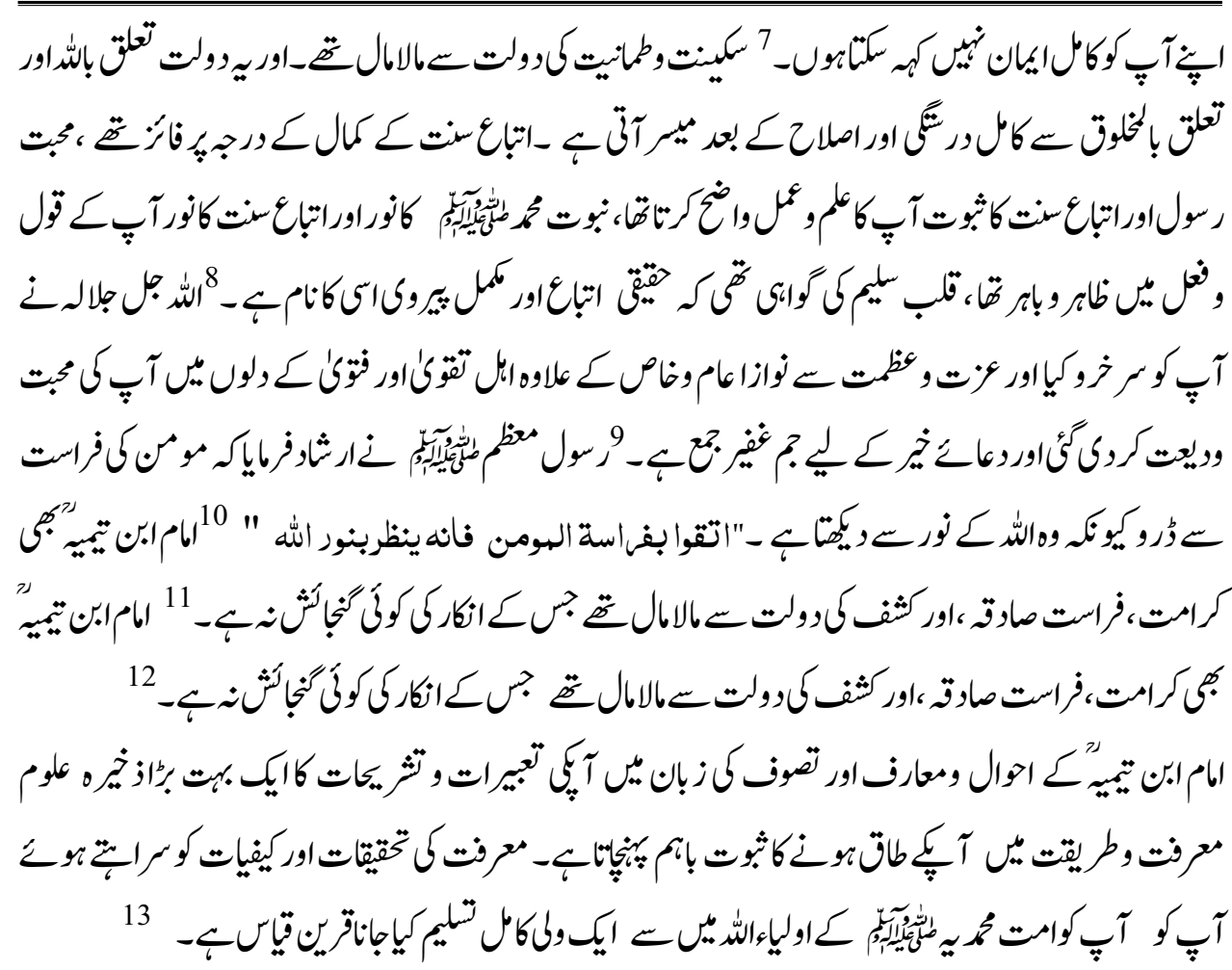

${ }^{7}$ Ibn Qayyim Jozi, Abu Abdullah Muhammad ibn Abu Bakr ibn Ayyub (691-751 AH). 1423 AH-2003, 1/520.

${ }^{8}$ Affandi, Alusi, Baghdadi, Khair-ud-Din Nu'man bin Mahmud (12521317 AH) Jala-ul-Ainin by the court of Ahmadin Ibn Taymiyyah. Ibn alHaytami, research: Al-Dani ibn Munir al-Zuhawi, modern library, Saida Beirut, Lebanon, I: 1, 2006. 1427 AH, p: 29.

${ }^{9}$ Nu'man ibn Mahmud, Jala 'al-Ainin, p. 25.

${ }^{10}$ Tirmidhi, Abu Isa Muhammad ibn Isa ibn Surah, Jami 'al-Tirmidhi, AlMizan, Urdu Bazaar, Lahore, Pakistan,145/2.

${ }^{11}$ Ibn Nasir al-Din Damascus Shafi'i (842 AH) Al-Rad al-Wafir, p: 251.

12 Ibn Nasir al-Din Damascus Shafi'i (842 AH) Al-Rad al-Wafir, p: 251.

${ }^{13}$ Ibn Qayyim Jozi, Madaraj Al-Salkin, 2/458. 


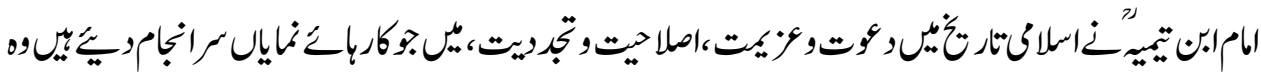

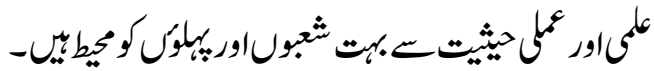

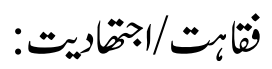

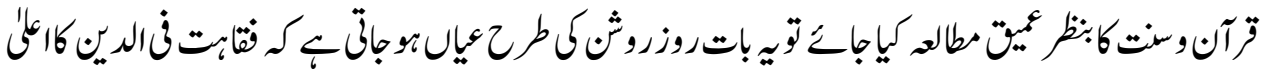

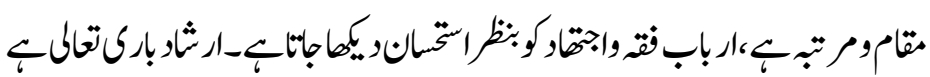

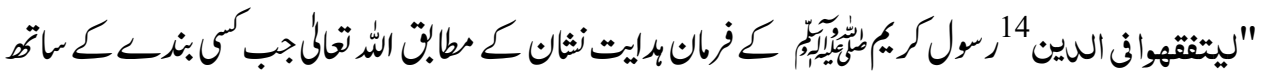
تي كارارهز

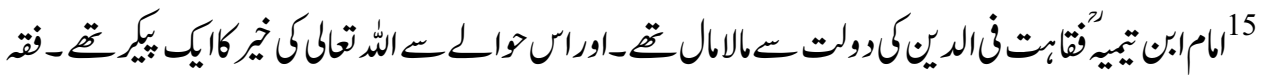

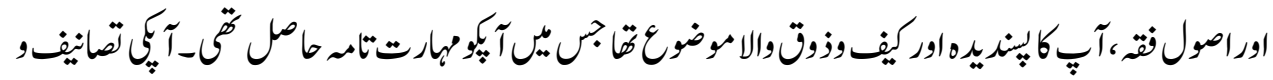

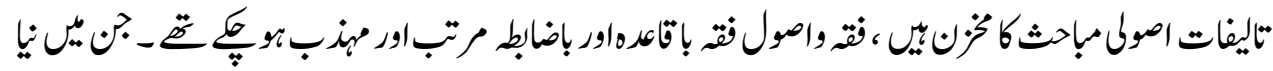

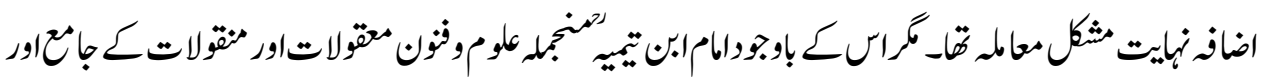

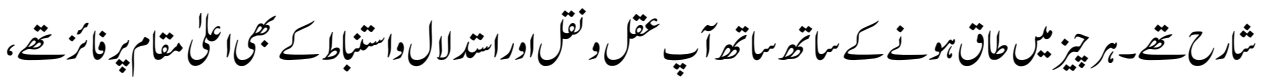

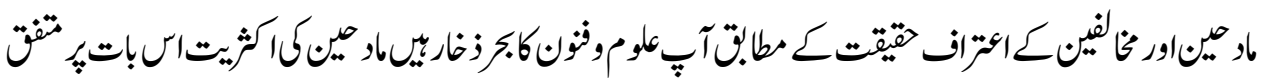

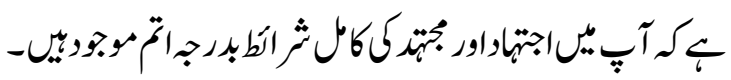

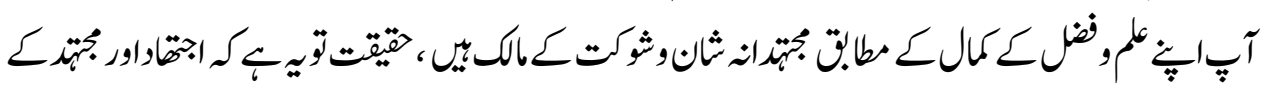

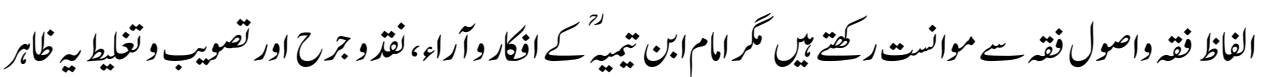

${ }^{14}$ Ibn Al-Imad, Shahab-ud-Din Abu Al-Falah Abdul Hayy Ibn Ahmad Ibn Muhammad Al-Askari Al-Hanbali Damascus (103-1089 AH) Damascus, Beirut, Lebanon, I: 1, 1406 AH. 1986, 8/137.

${ }^{15}$ Ali Qari, Ali Ibn Sultan Mahmud (1014 AH) Marqaat Al-Mufatih Sharh Mishkat Al-Masabih, Research: Jamal Aishani, Dar Al-Kitab Al-Alamiya Beirut, Lebanon 2001 Kitab al-Labas, 8/216. 


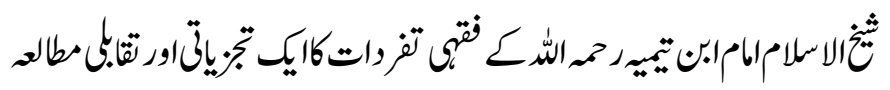

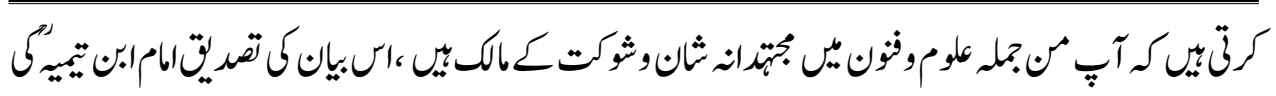

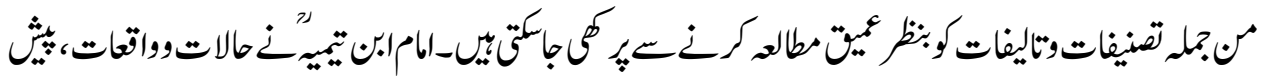

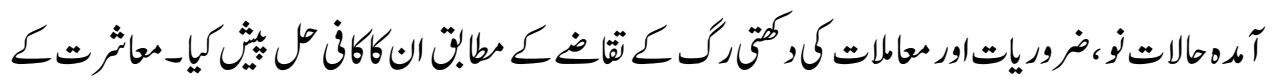

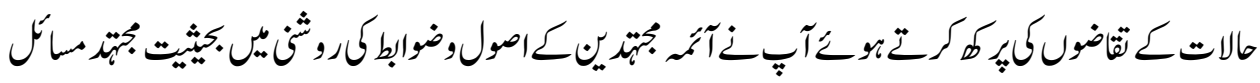

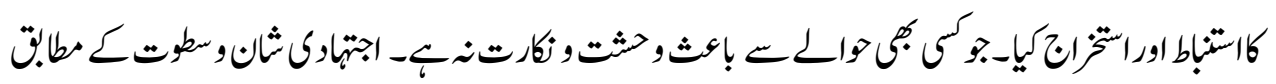

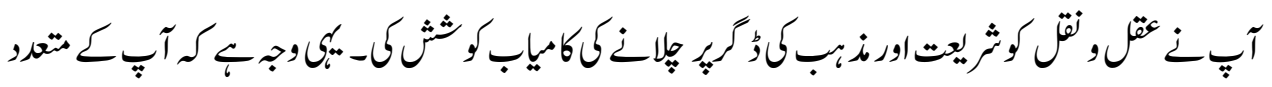

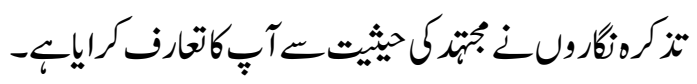

نغنجواسلوب:

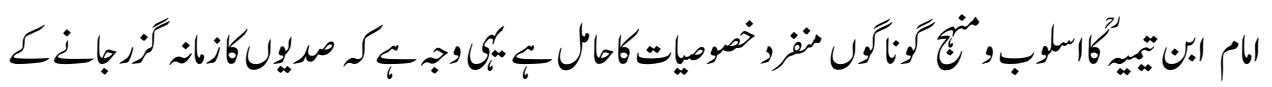

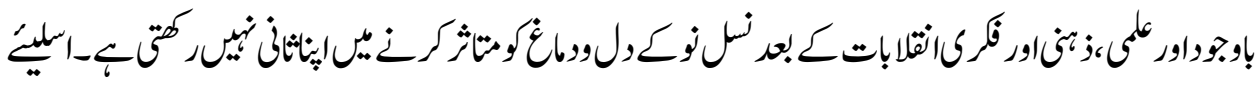

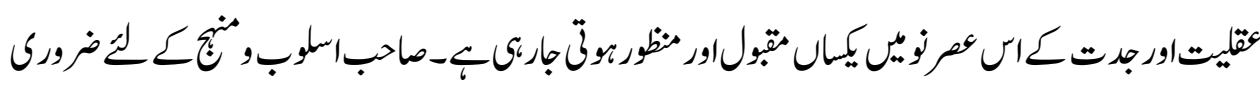

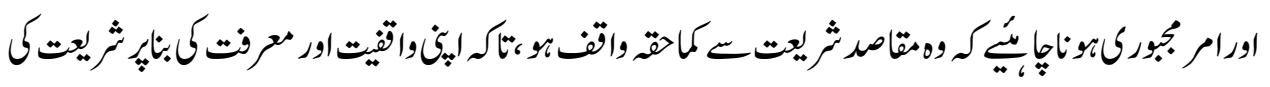

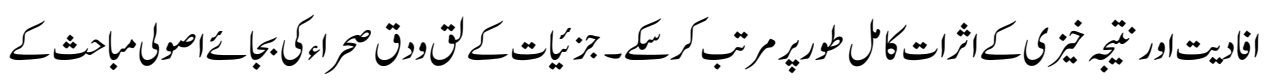

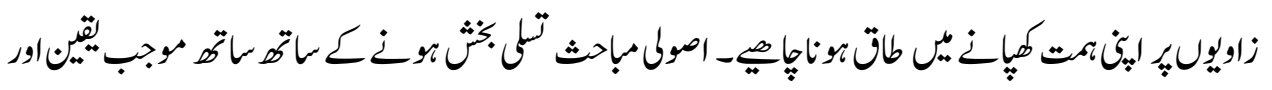

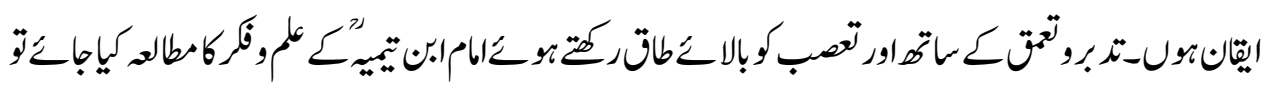

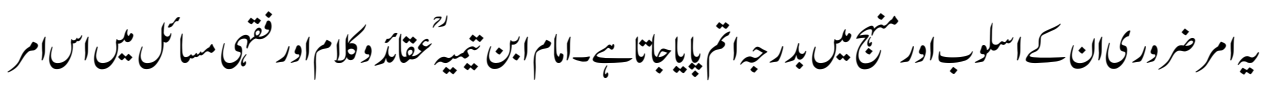

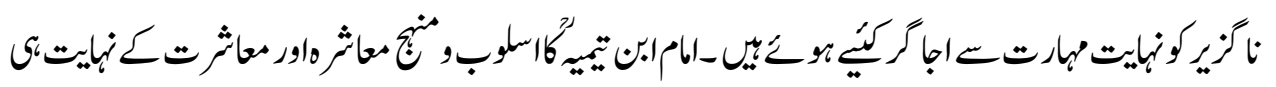

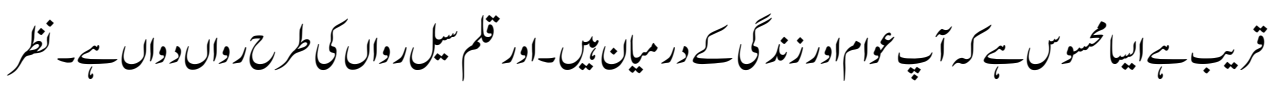

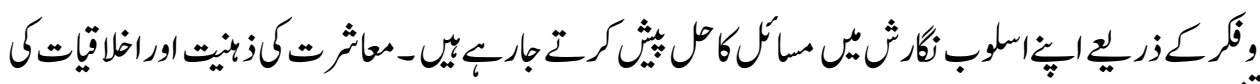

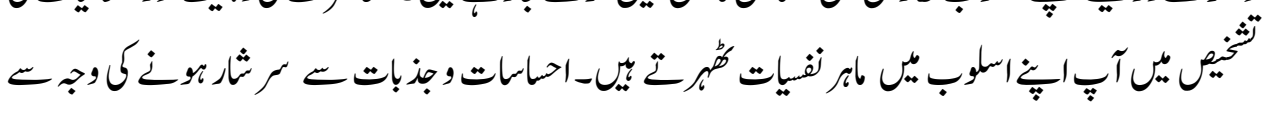

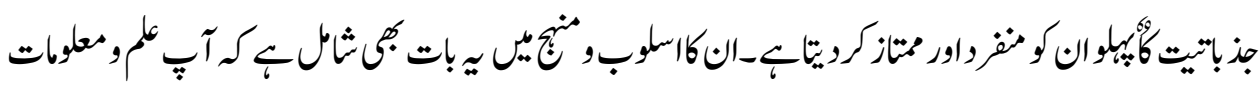

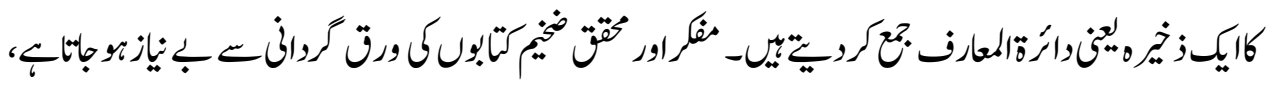

27 


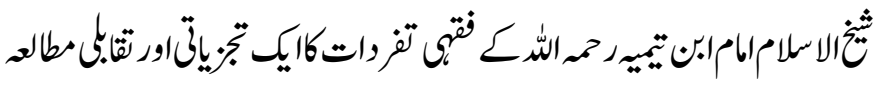

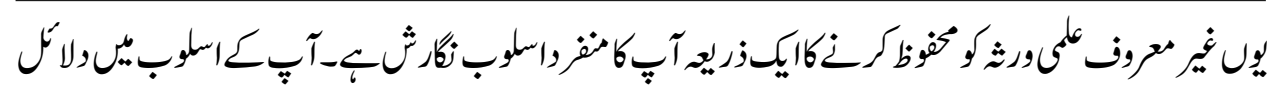

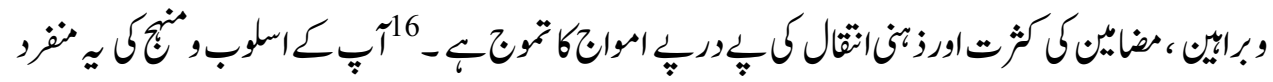

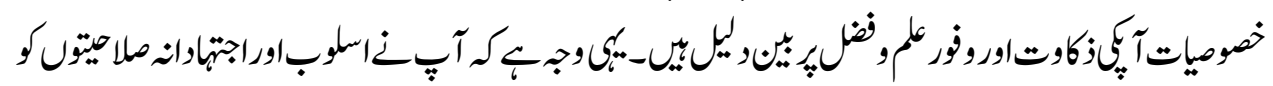

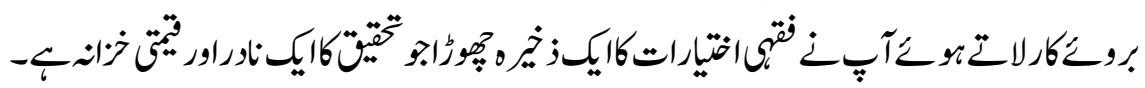

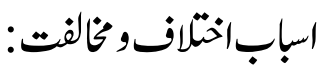

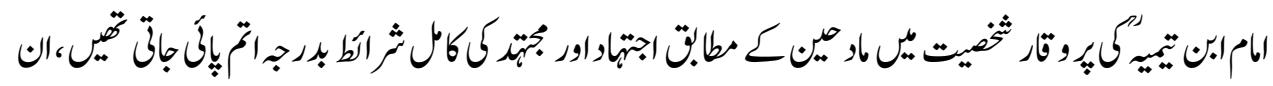

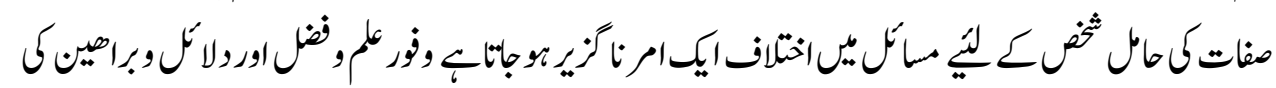

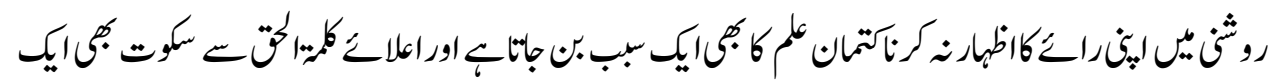

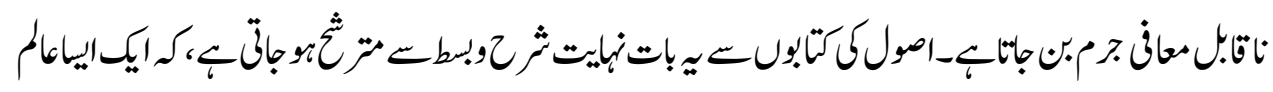

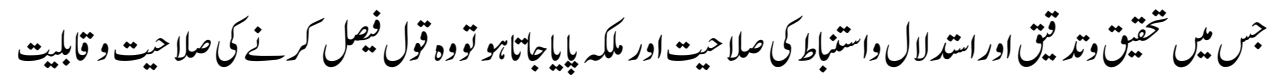

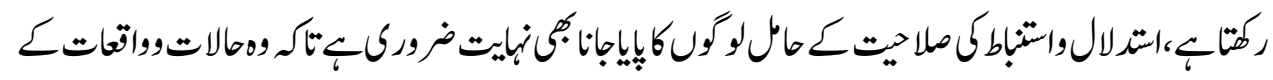

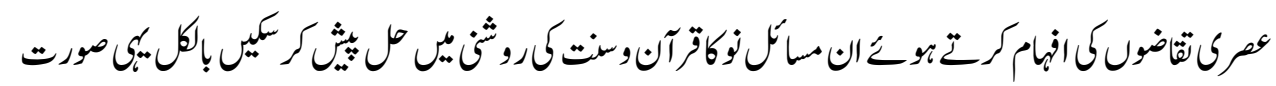

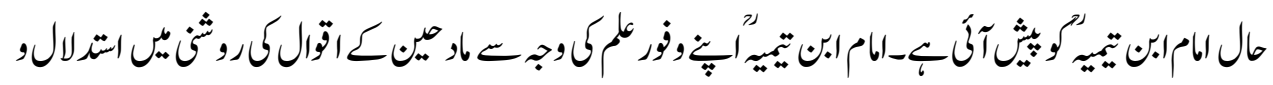

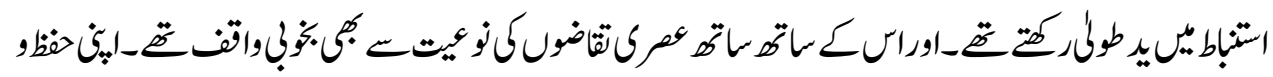

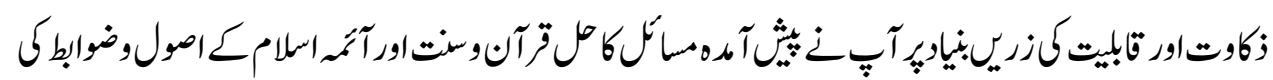

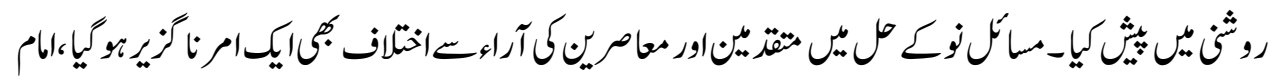

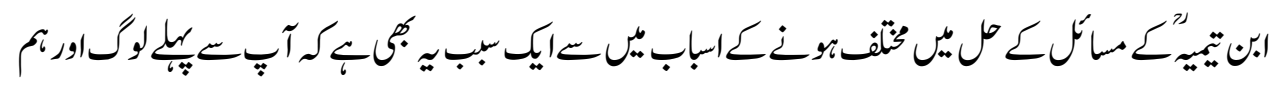

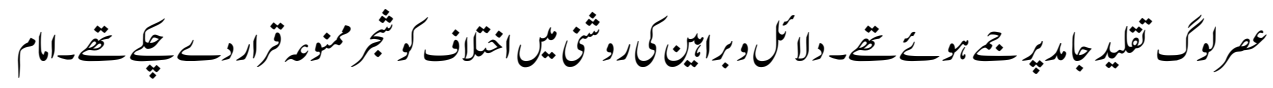

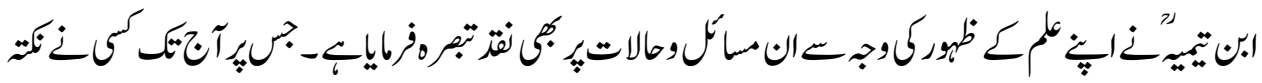

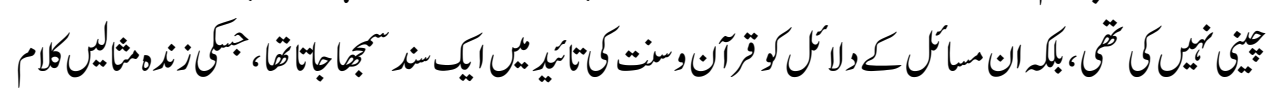

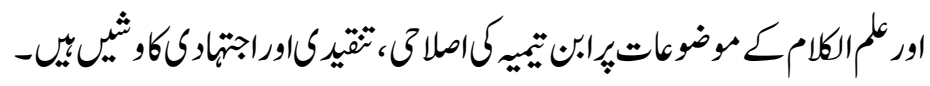

${ }^{16}$ Quran, repentance (9) 122. 


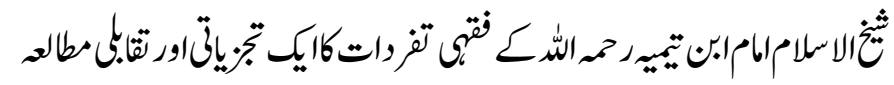

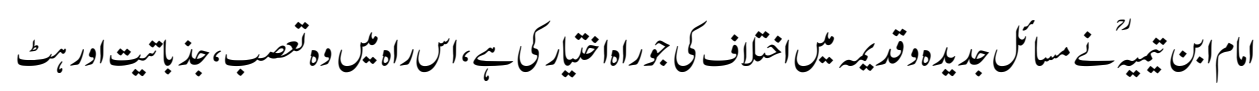

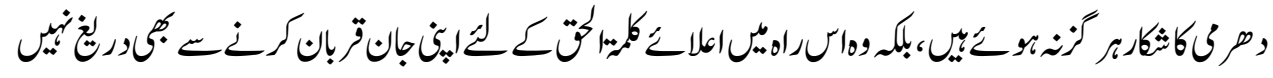

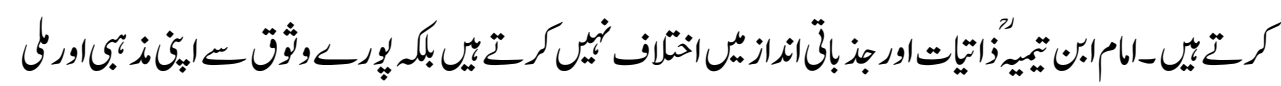

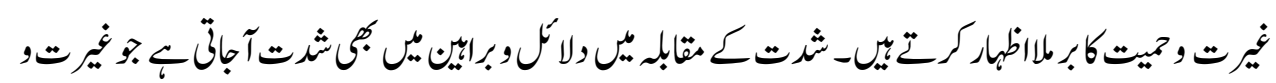

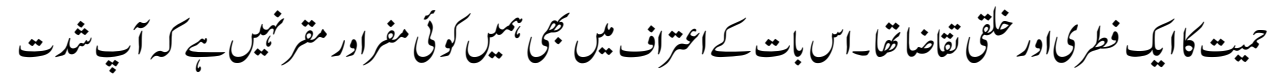

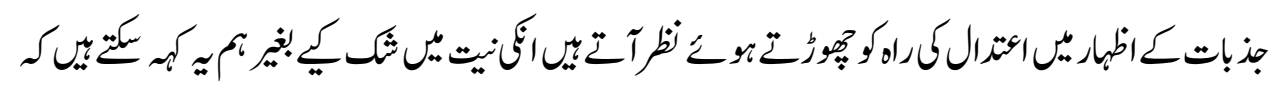

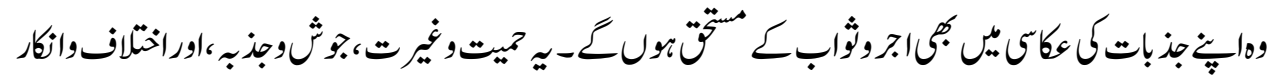

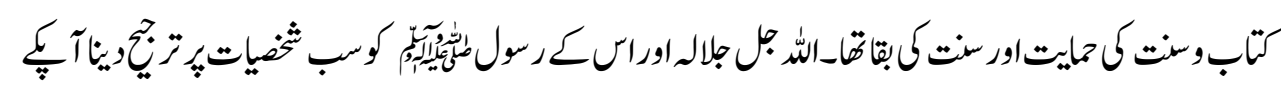

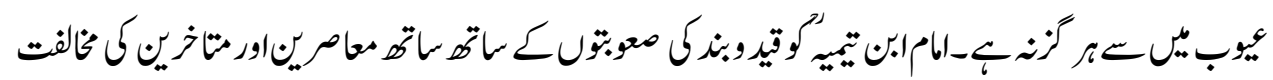

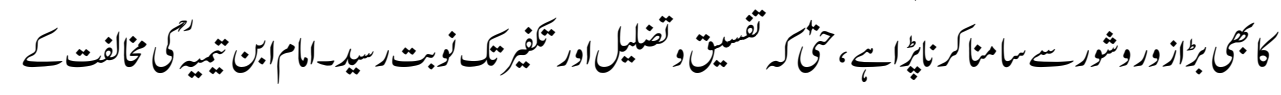

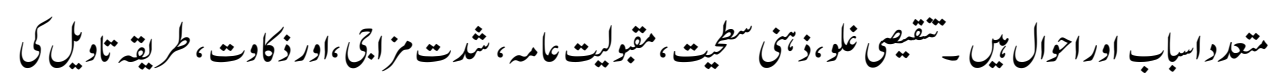

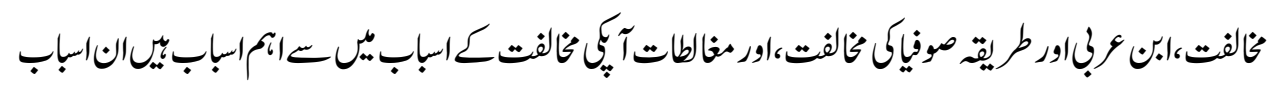

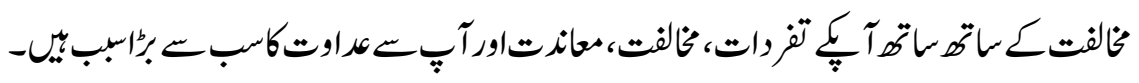
تز وات:

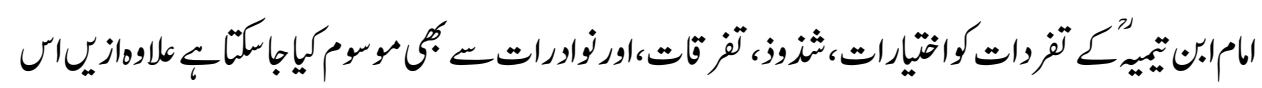

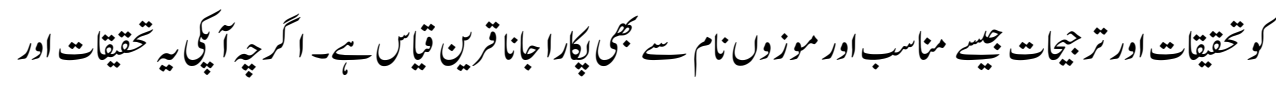

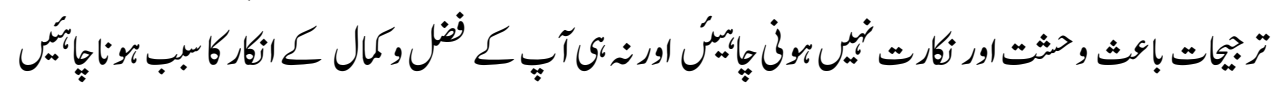

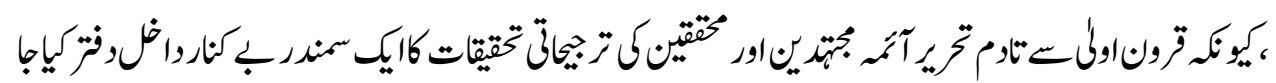

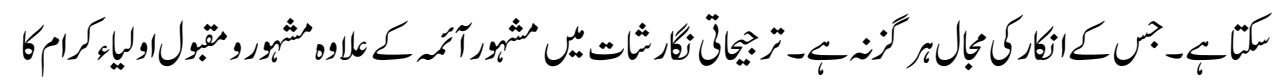

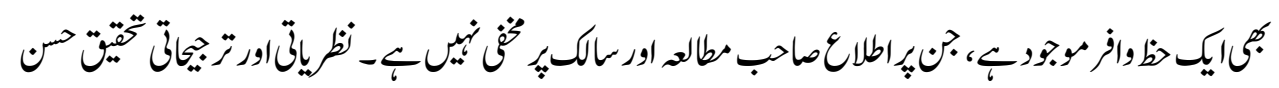

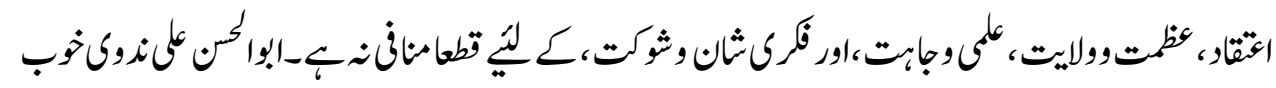
-

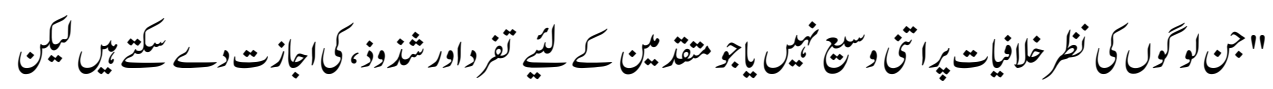

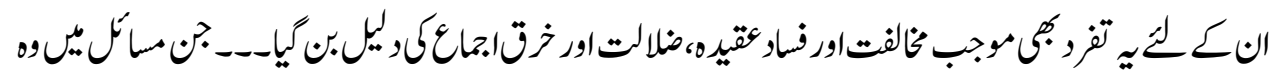




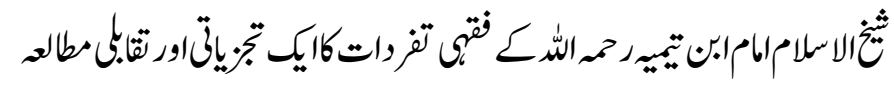

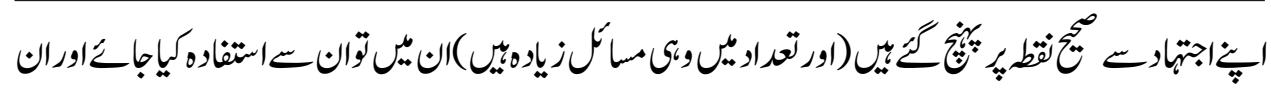

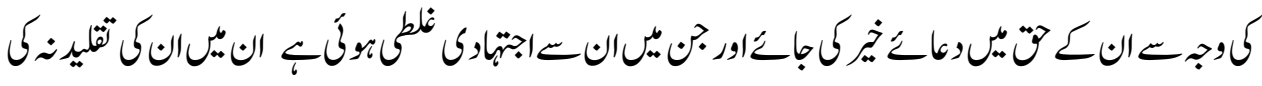

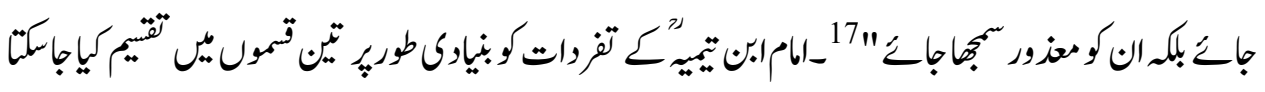

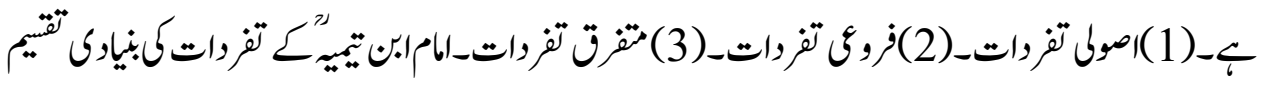

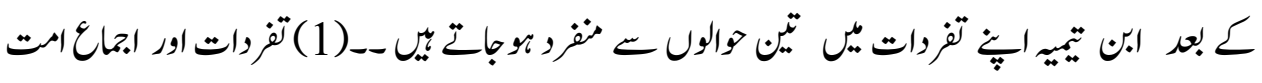

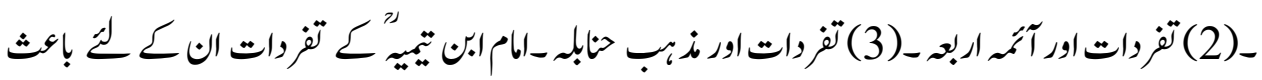

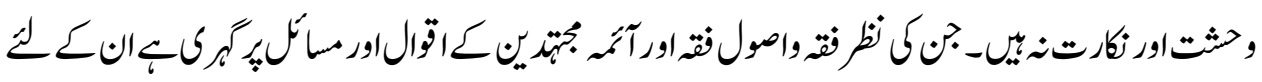

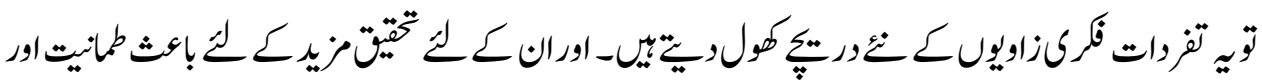

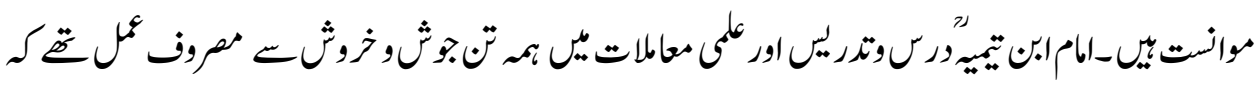

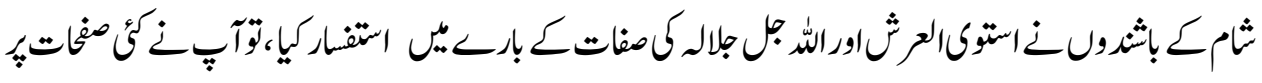

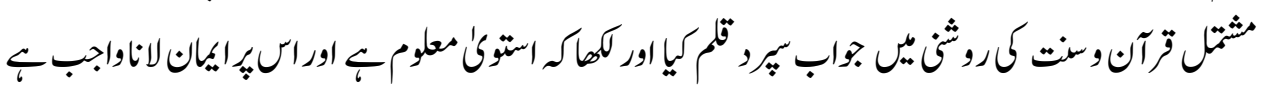

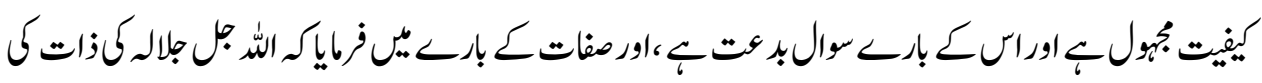

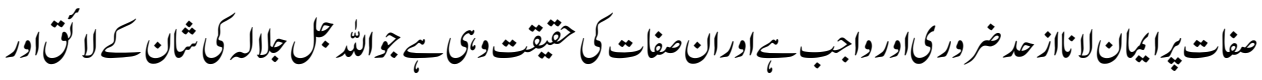

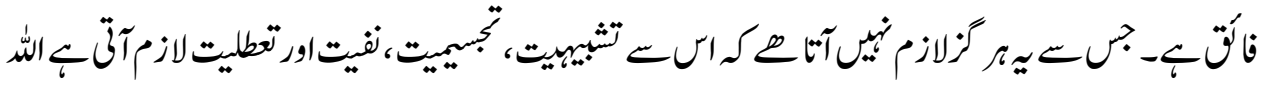

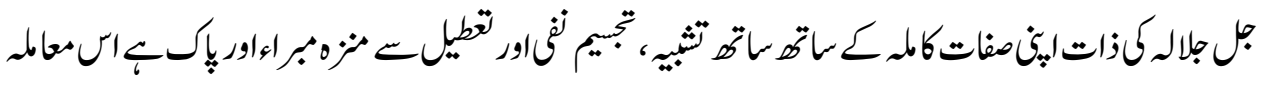

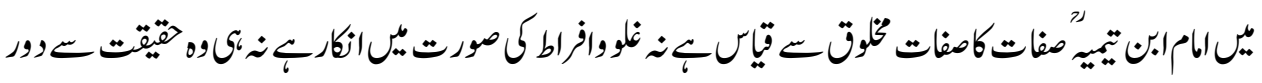

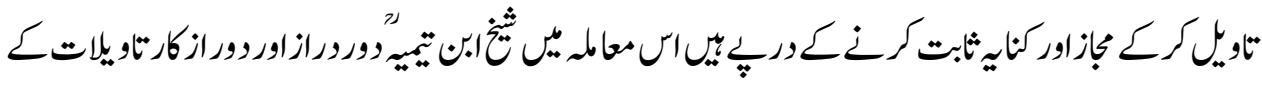

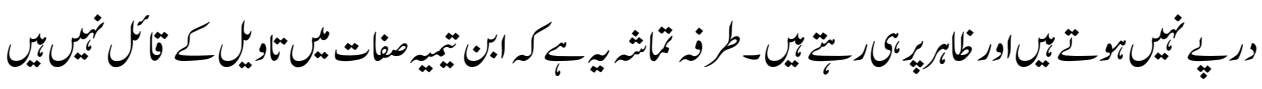

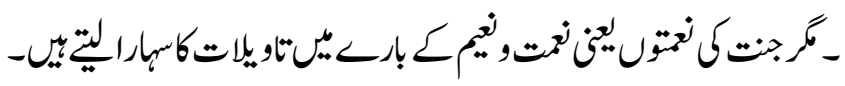

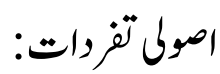

${ }^{17}$ Hasani, Nazhat al-Khawatir, degree of translation by Muhammad bin Abdul Rahim Al-Armawi, 2/201 


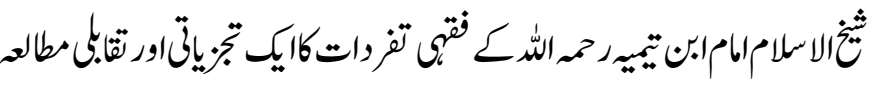

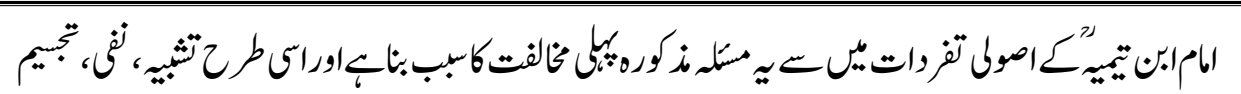

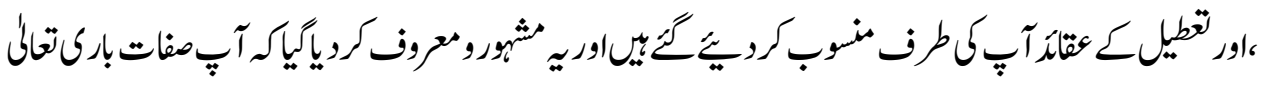

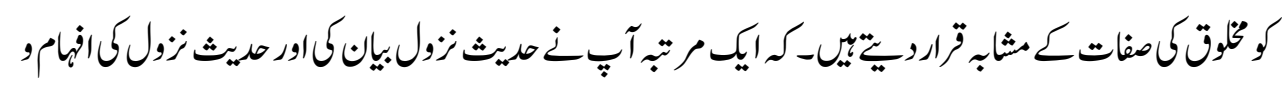

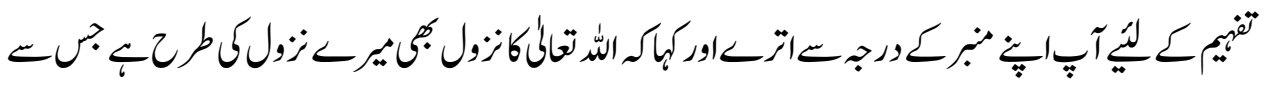

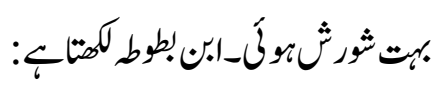

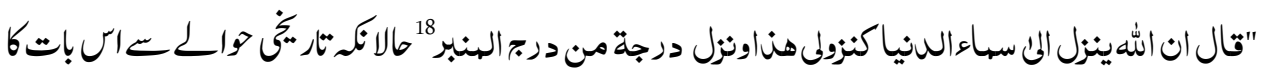

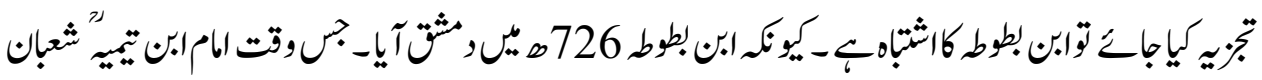

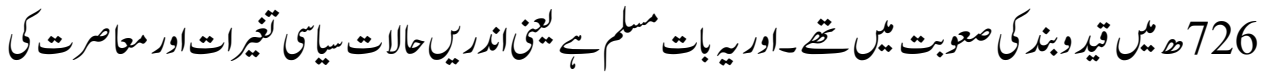

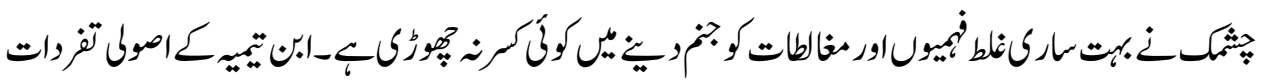

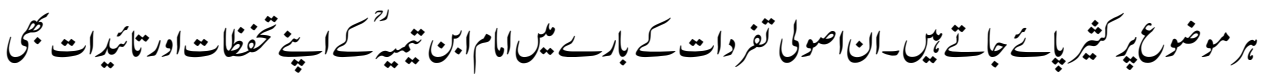

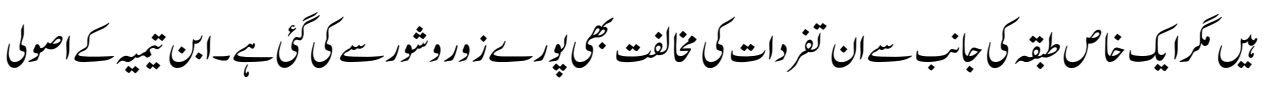

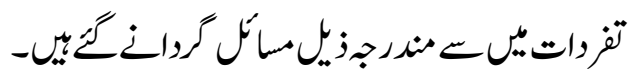

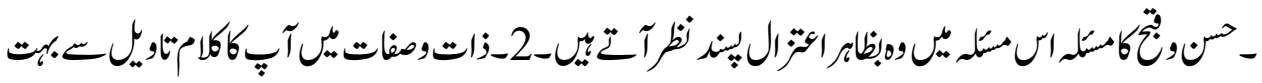

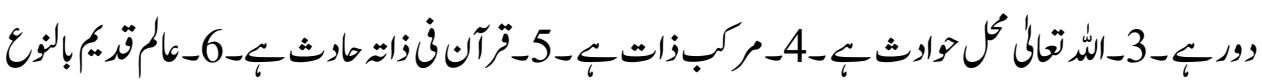

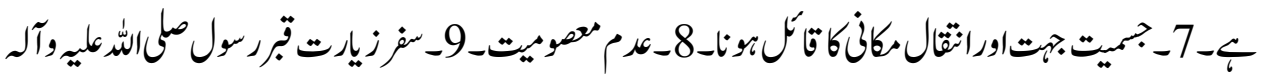

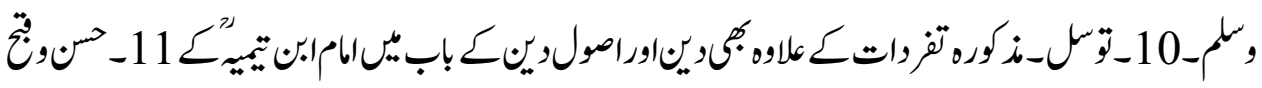

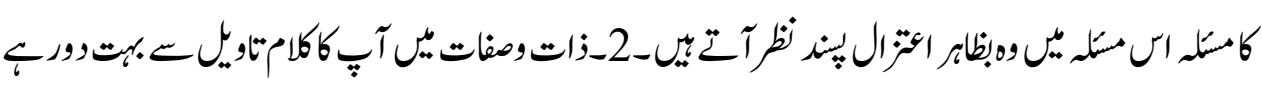

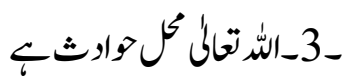

18. Ibn Battuta, The Journey of Ibn Battuta, "The Gift of Sight in the Strangers and the Wonders of Travel" 1987, 1/111. 


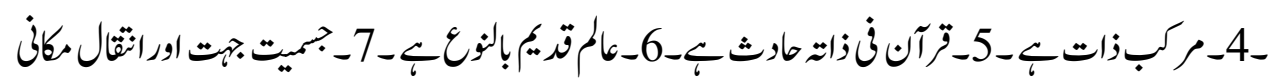

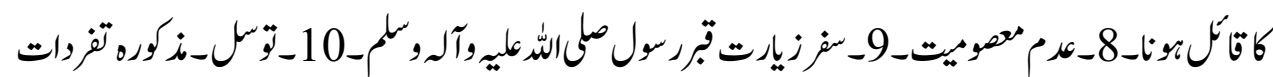

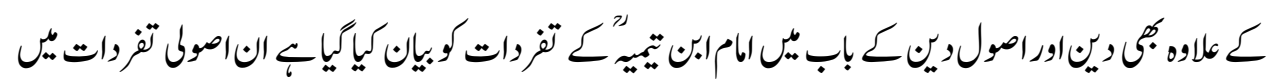

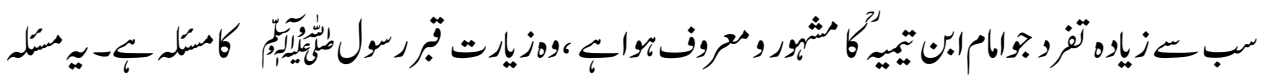

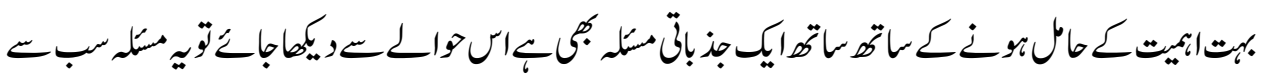

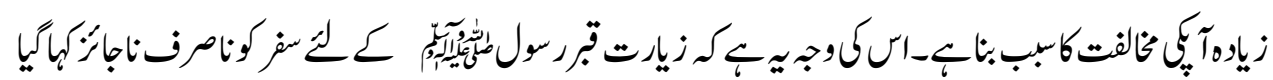

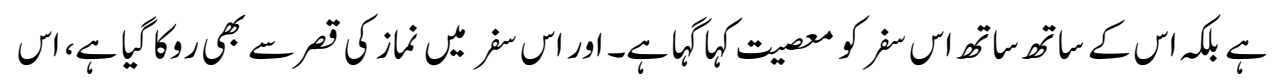

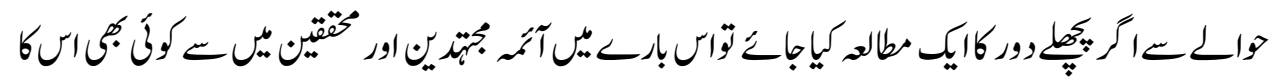

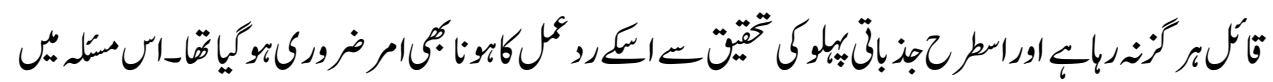

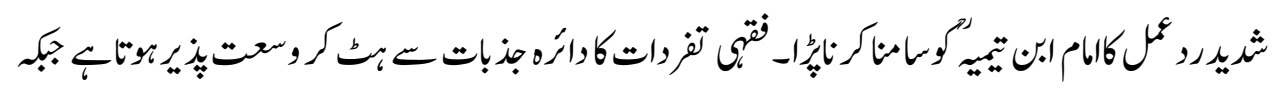

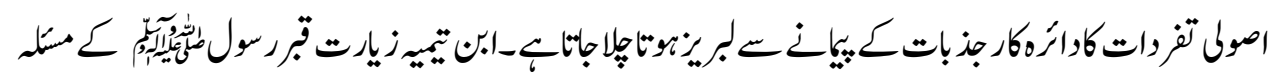

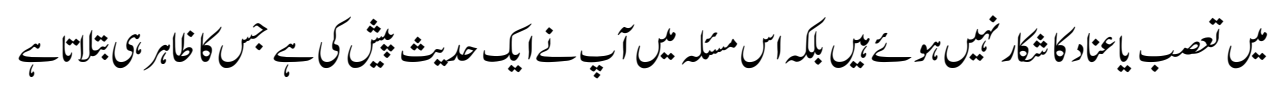

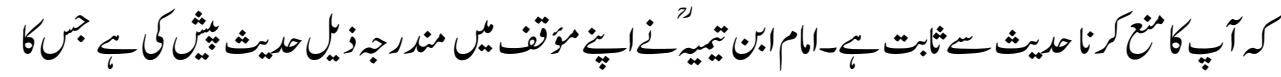

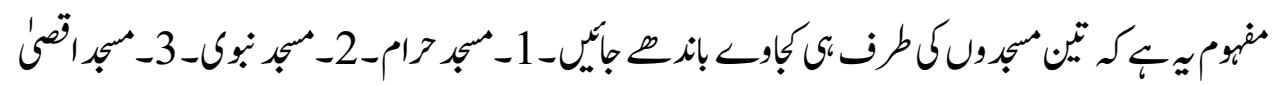

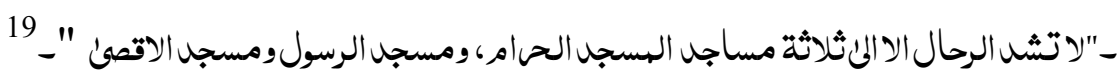

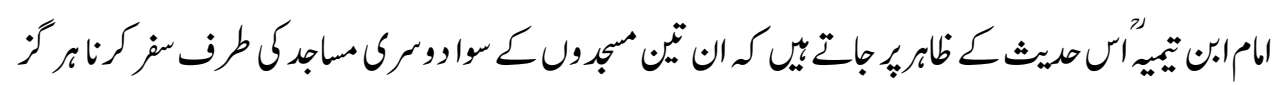

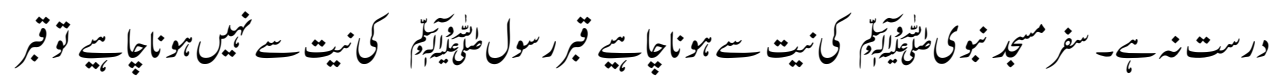

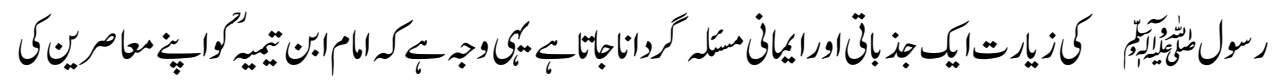

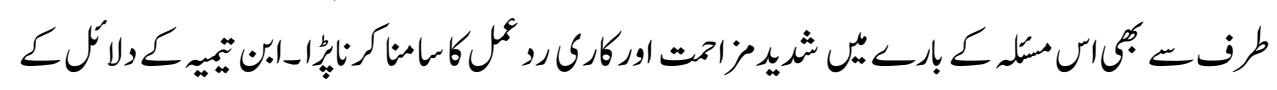

19) Bukhari, Muhammad bin Ismail, Al-Sahih Al-Bukhari, Ancient Library, Karachi, Pakistan, No. 1/58. 


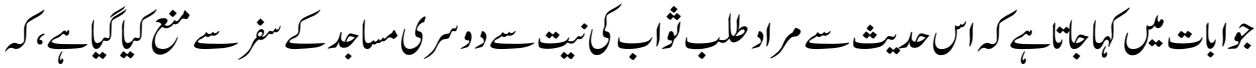

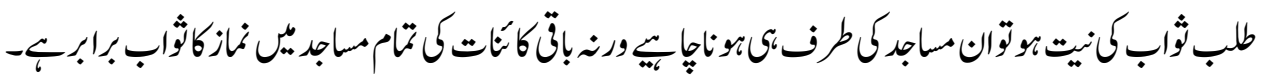

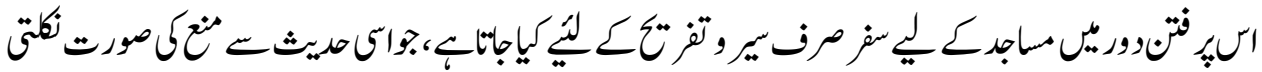

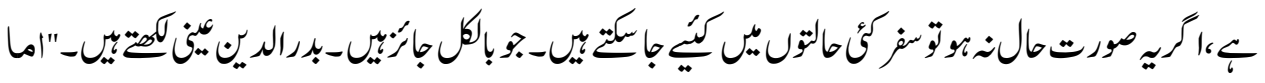
تصن غير الساجل من الرحلة فنلب العلم ونى التجارة وزياة الصالحين والشاهل وزيارة الاخوان ونحو

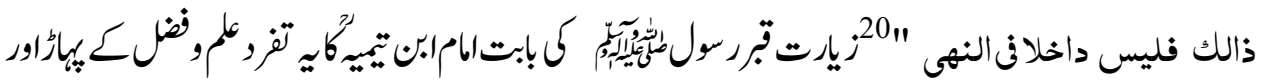

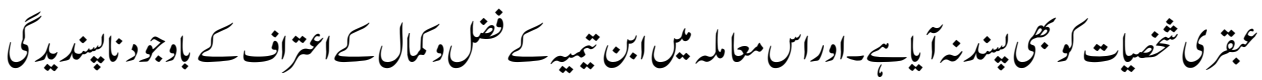

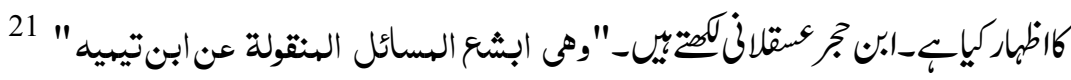

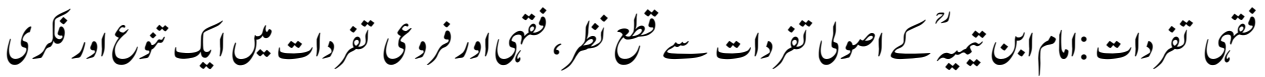

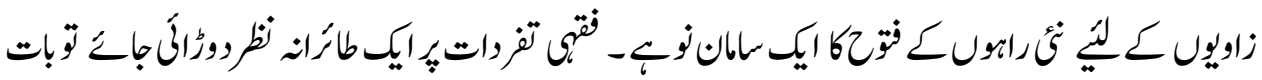

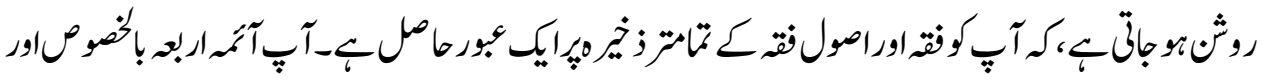

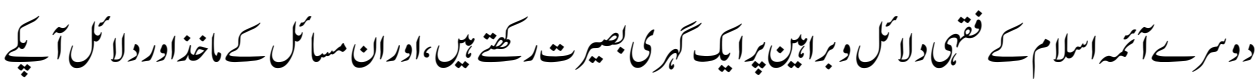

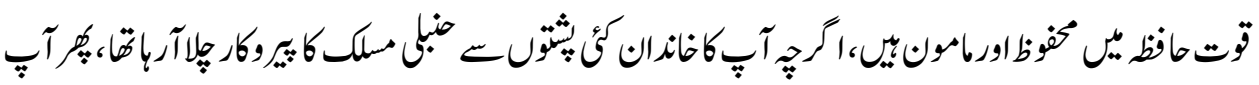

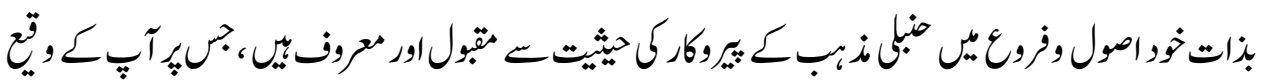

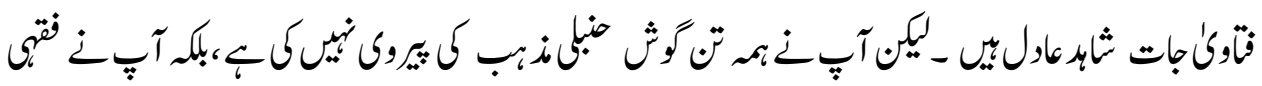

${ }^{20}$ Aini, Badruddin, Abu Muhammad Mahmud bin Ahmad (855 AH) Umdat al-Qari Sharh Sahih Bukhari, Seizure and Correction: Abdullah Mahmud,Muhammad Umar Dar Al-Kitab Al-Alamiya, Berurat, Lebanon, I: 1, 1421 AH. 2001, Book of Fazal Salat in the Mosque of Mecca and Medina, 7/370.

${ }^{21}$ Fateh Al-Bari Sharh Sahih Al-Bukhari, Asqalani, Ahmad Ibn Ali Ibn Hajar (773-852 AH) Research and Commentary: Abdul Qadir Shaybah AlHamd Maktab Al-Mulk Fahd, Riyadh, Arab, I: 1, 1421 AH. 2001/3/79. 


\section{The Scholar Islamic Academic Research Journal}

Vol. 6, No. 2 || July-December 2020 || P.20-42

https://doi.org/10.29370/siarj/issue11urduar2

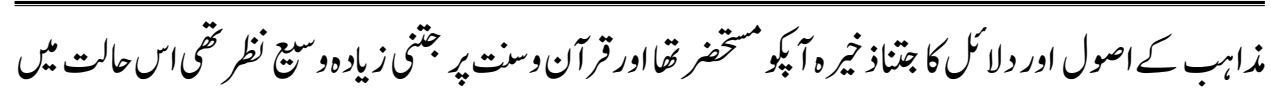

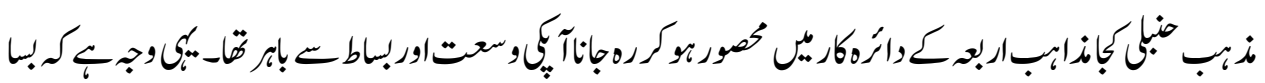

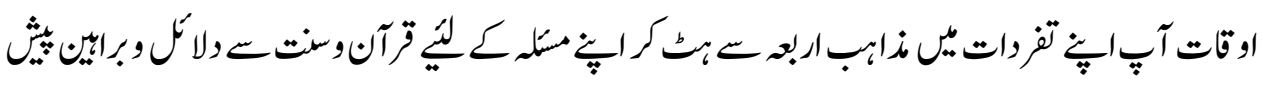

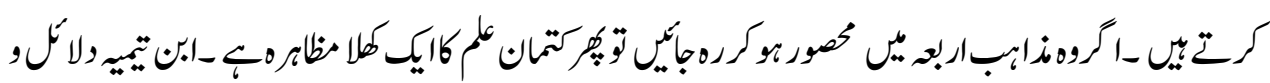

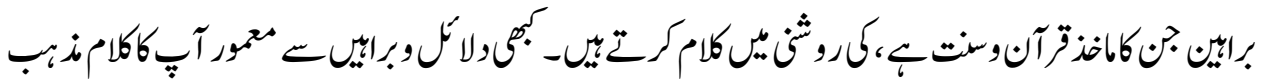

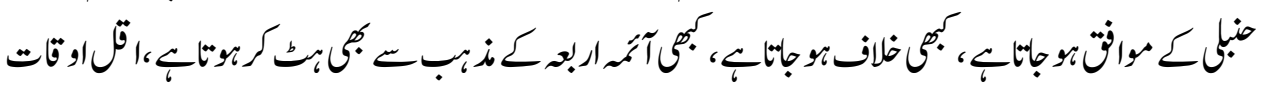

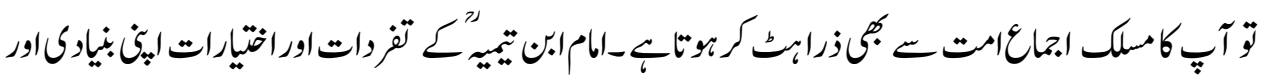

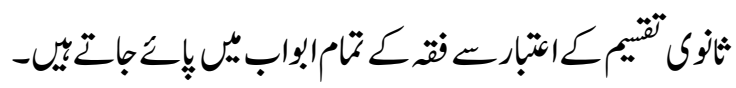

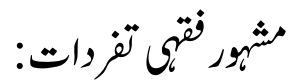

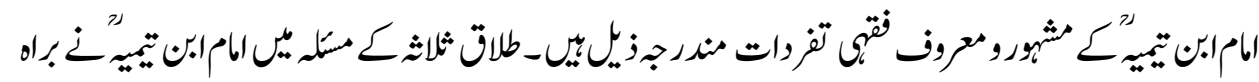

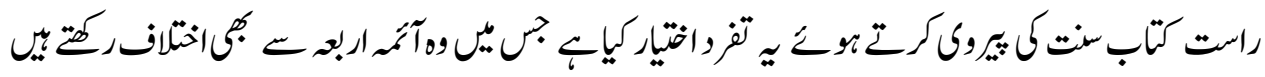

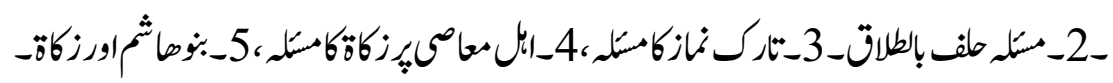

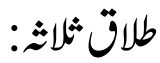

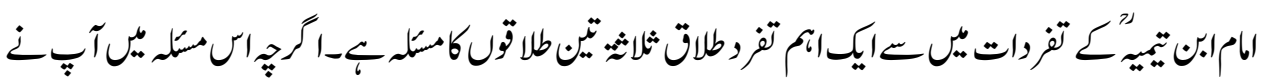

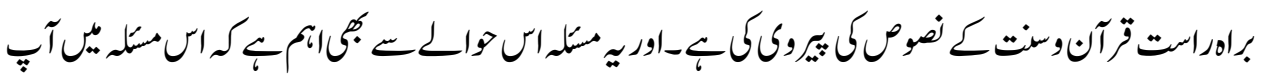

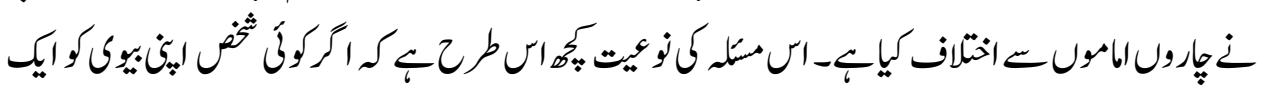

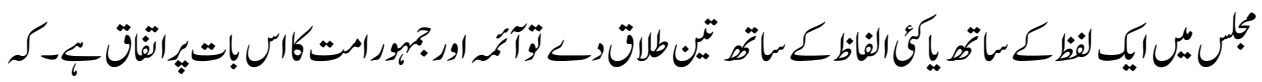

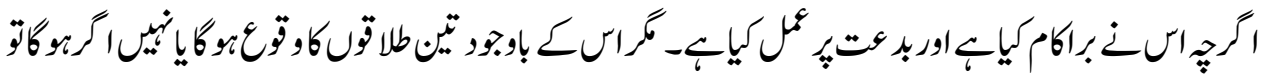

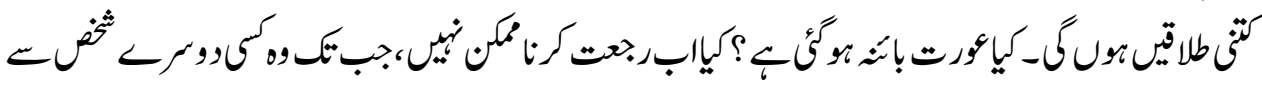

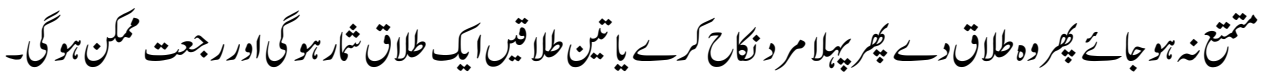

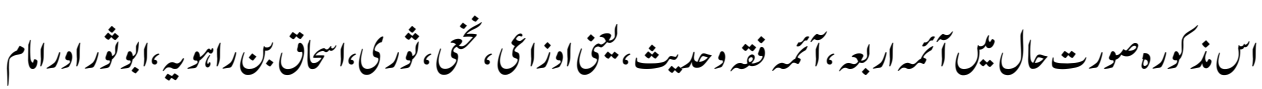

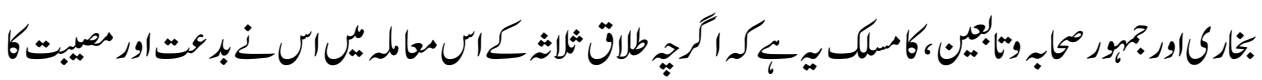

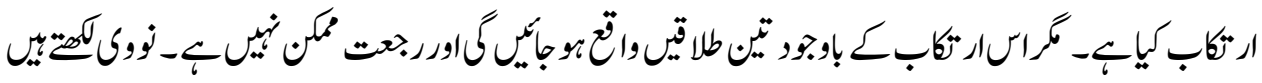




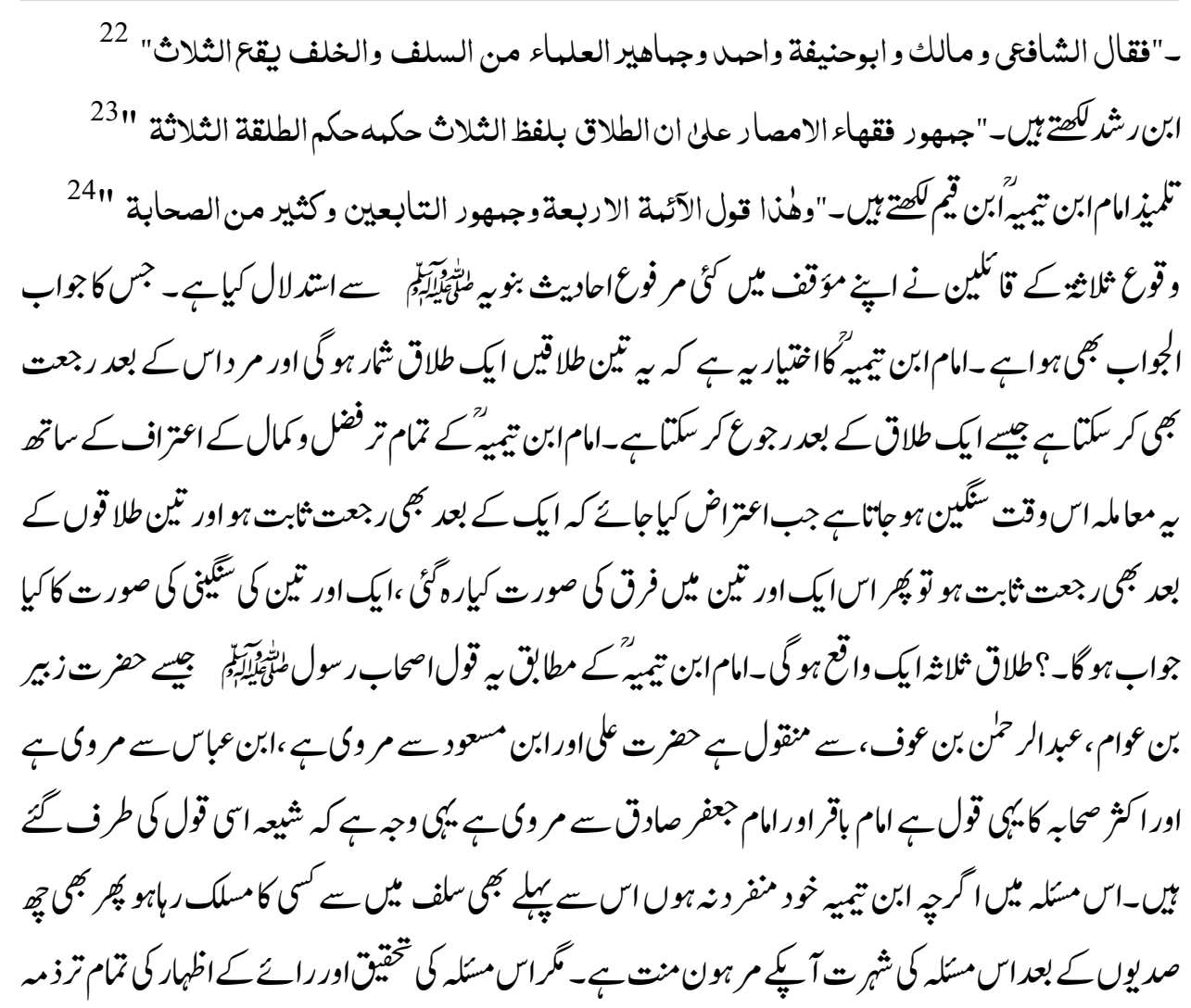

${ }^{22}$ Nawawi, Sahih Muslim Bashar H Al-Nawawi, Al-Mutti'at Al-Masriyat, Al-Azhar, Egypt, I: 1, 1347 AH. 1929 10/70.

23 Ibn Rushd, Muhammad ibn Ahmad ibn Ahmad ibn (Muhammad) Ahmad ibn Ahmad ibn Rushd, Sharh al-Bada'at al-Mujtahid wa Nahayyat al-Muqtasid wa Baha Musha al-Sabeel al-Murshid, Sharh wa Tahaqiq wa Takhrij: Abdullah al-Abadi, Dar al-Islam 1, 1416 AH-1995 AD, 3/1381.

${ }^{24}$ Ibn Qayyim Jozi, Abu Abdullah Muhammad ibn Abu Bakr ibn Ayub (691-751 AH) Zad al-Ma'ad fi Huda Khair al-Ibad, Research: Ali ibn Muhammad Imran, Muhammad Aziz Shams, Graduation: Ibrahim ibn Muhammad Shibli, Arab, I: 1, 1439 AH. 2018, 5/353. 
The Scholar Islamic Academic Research Journal

Vol. 6, No. 2 || July-December 2020 || P.20-42

https://doi.org/10.29370/siarj/issue11urduar2

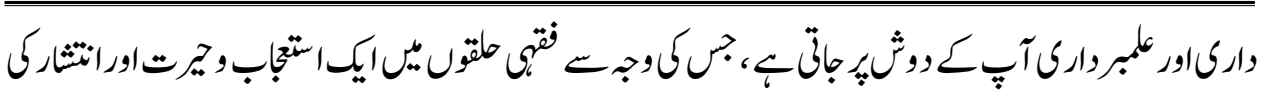

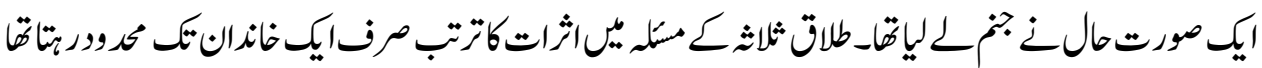

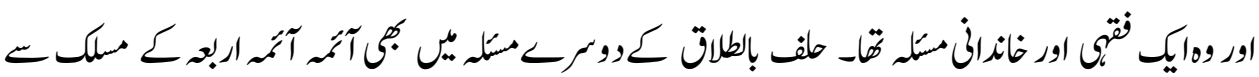

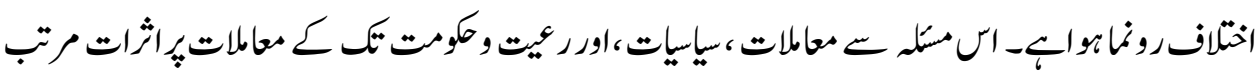

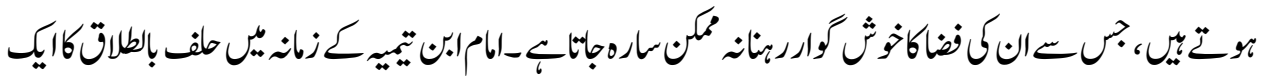

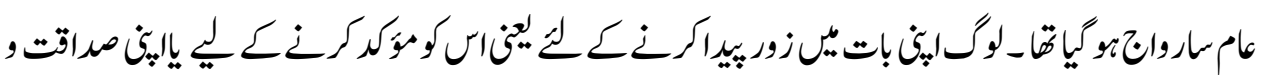

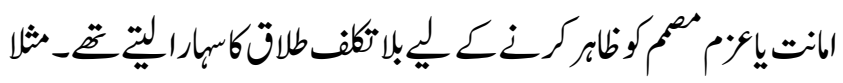
"على الطلاق لافعدن كنا ، على الطلاق لامتنعن عن كنا ، على الطلاق لتفعلن كنا ـ اورعلى الطلاق

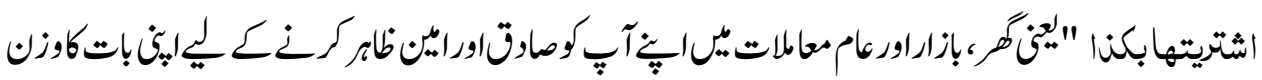

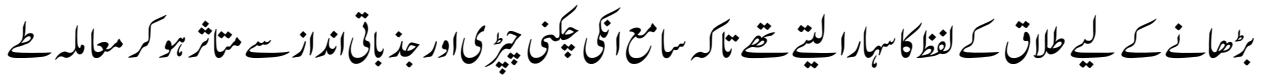

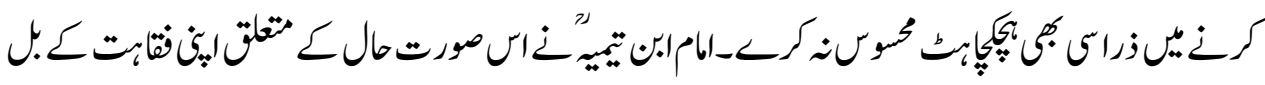

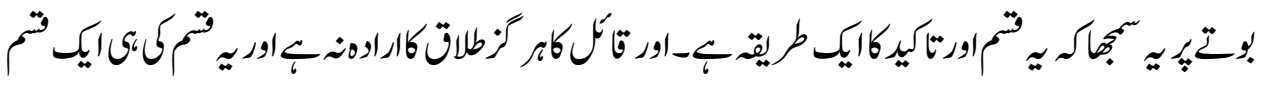

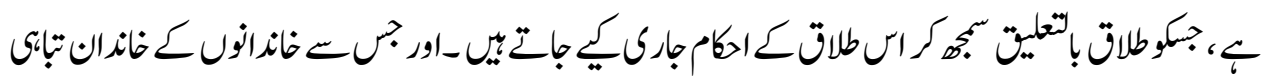

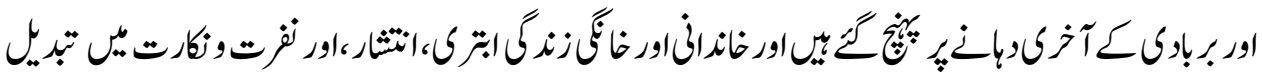

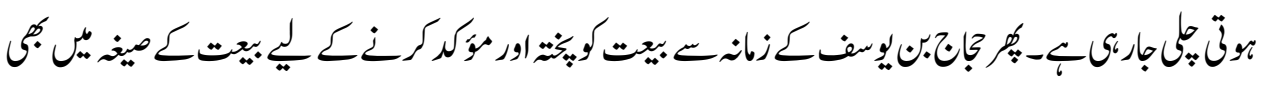

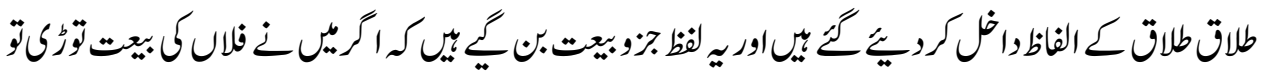

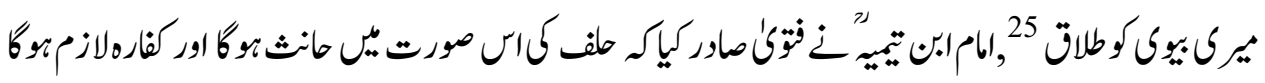

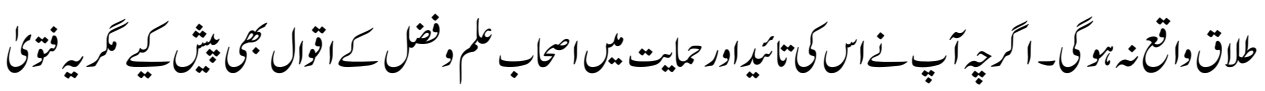

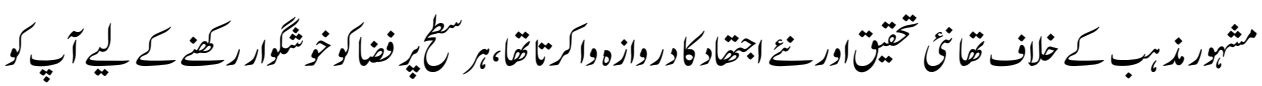

${ }^{25}$ Nadwi, Sayyid Abu Al-Hassan, History of Da'wah and Commitment, $1 / 106$. 


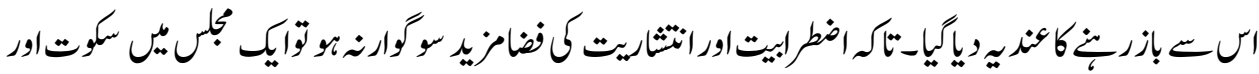

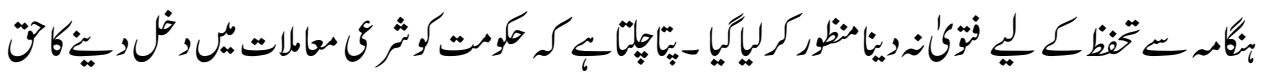

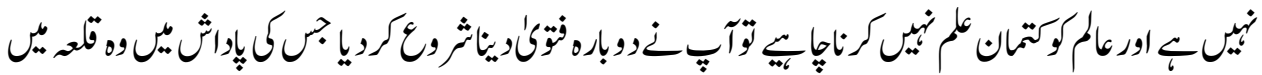

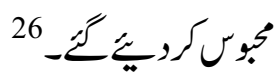

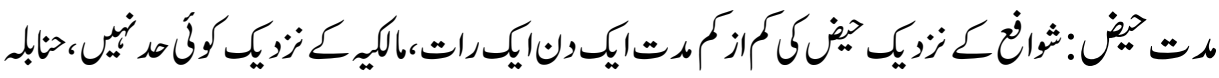

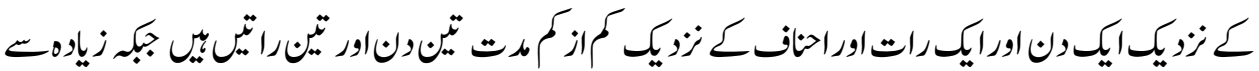

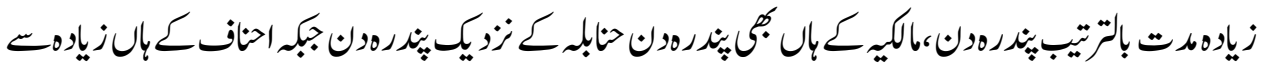
زيايرهد

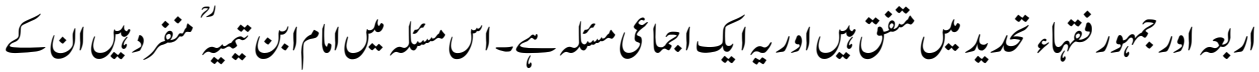

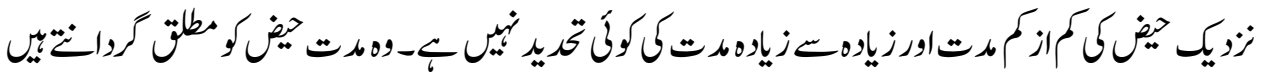

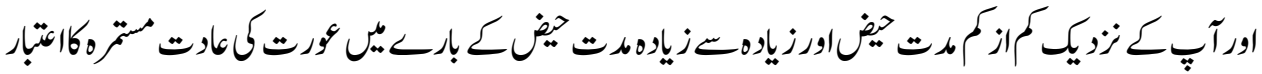

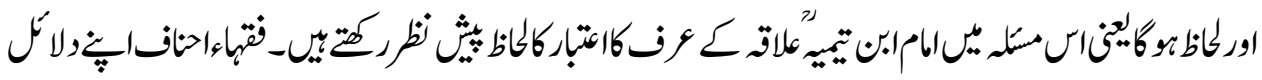

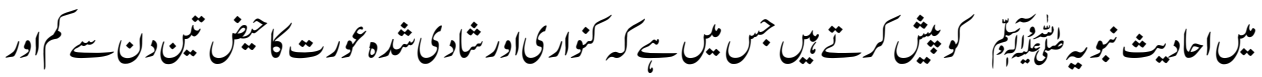

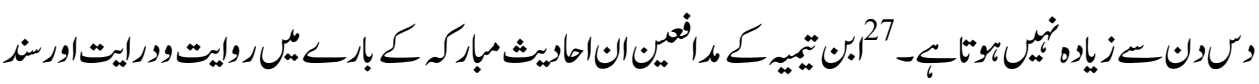

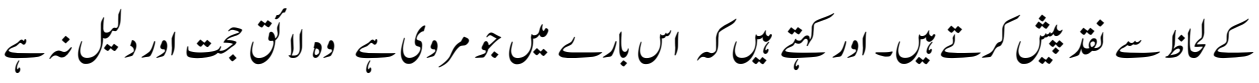

${ }^{26}$ Ibn Kathir, The Beginning and the End, 16/148.

${ }^{27}$ Dar al-Qatani, Ali ibn Umar (385 AH) Sunan al-Dar al-Qatani, research and suspension: Adil Ahmad Abdul Mawjood, Ali Muhammad Masoos, Dar al-Ma'rifah, Beirut, Lebanon, I: 1, 1422 AH. 2001, 1/484. Hadith number: 833. 


\section{The Scholar Islamic Academic Research Journal}

Vol. 6, No. 2 || July-December 2020 || P.20-42

https://doi.org/10.29370/siarj/issue11urduar2

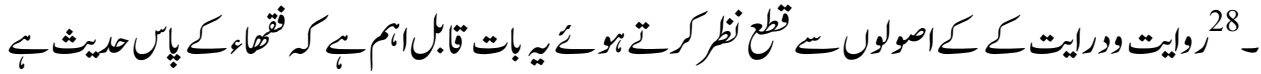

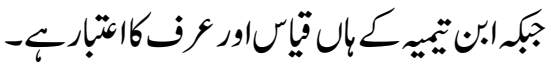

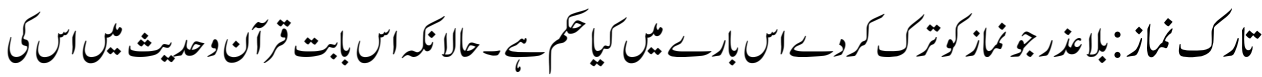

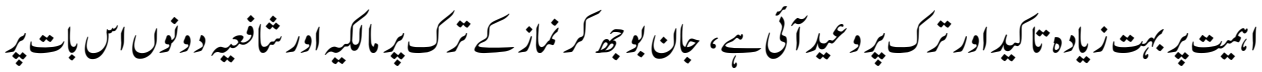

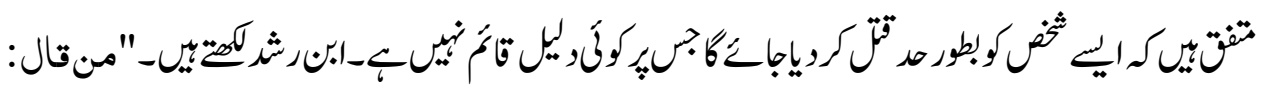

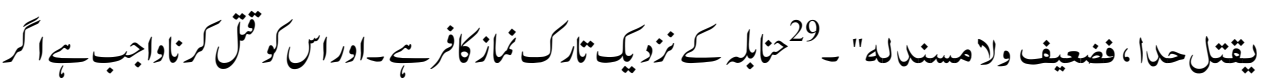

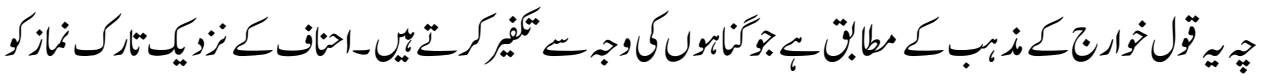

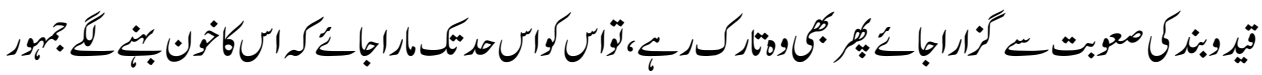

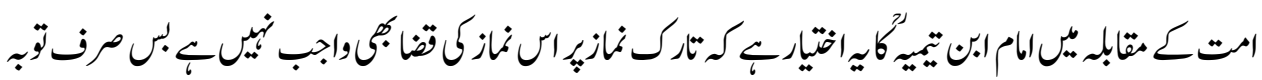

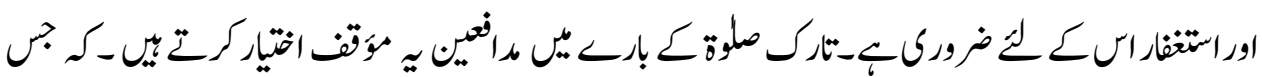

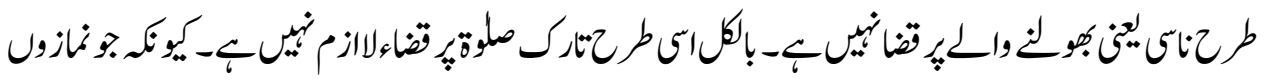

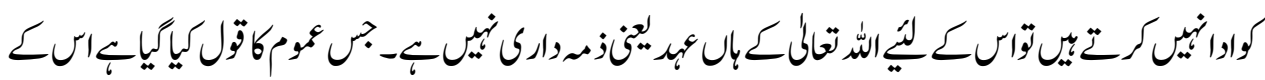

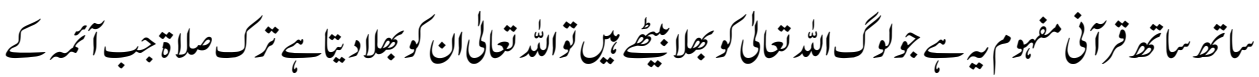

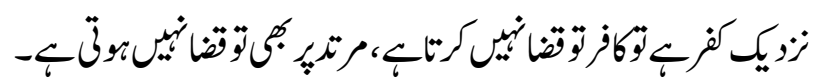

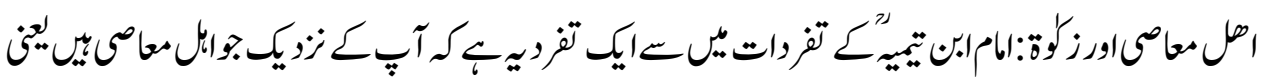

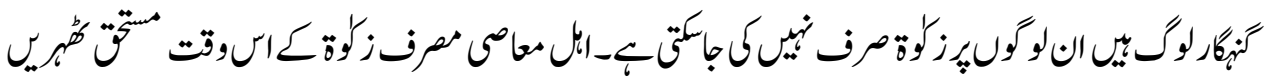

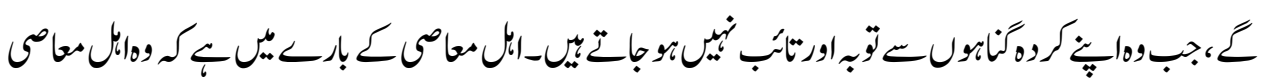

${ }^{28}$ Qanoji, Bhopali, Abul Tayyab Siddiq bin Hassan bin Ali Hussaini Bukhari, Al-Rawdat Al-Nadia, Sharh Al-Darr Al-Bahiyat, Ancient Library, Karachi, Pakistan, S.N. 1/62.

${ }^{29}$ Ibn Rushd, Bada'at al-Mujtahid, 1/217. 


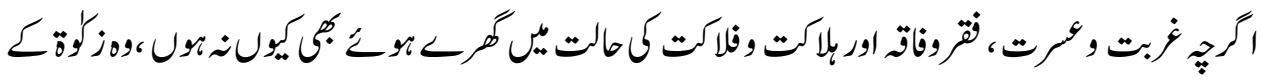

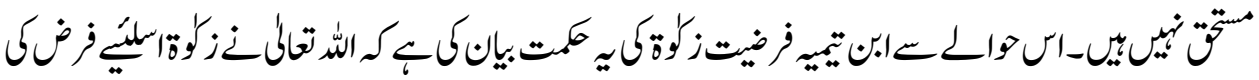

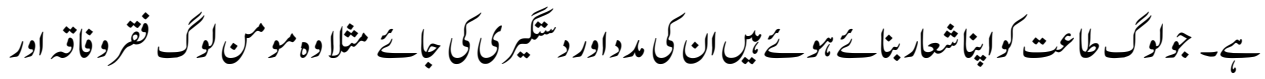

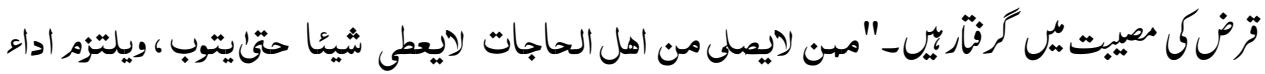

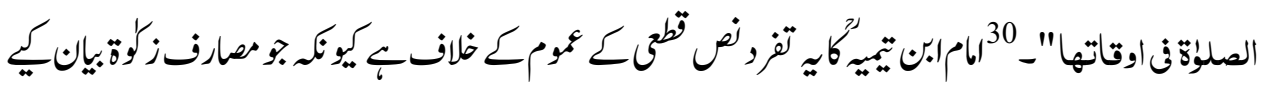

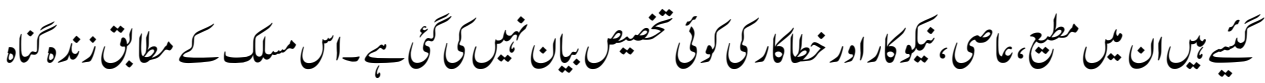

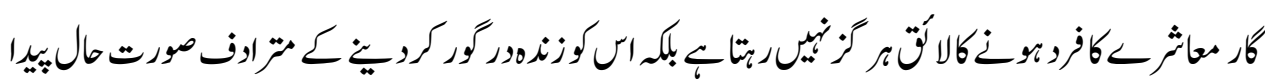

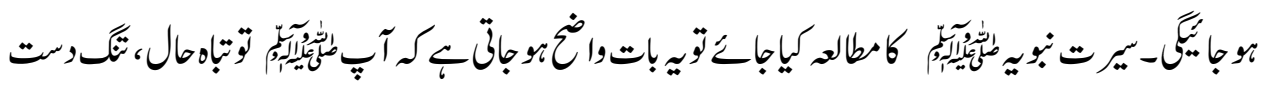

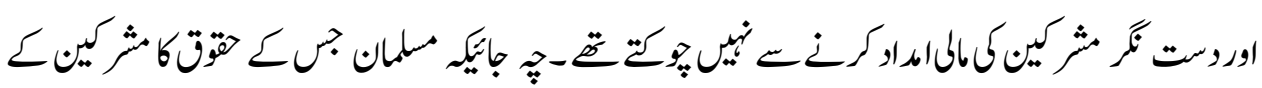

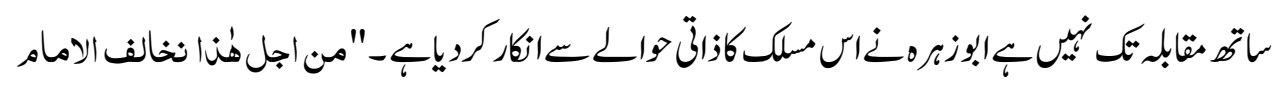

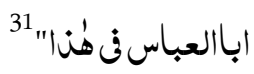

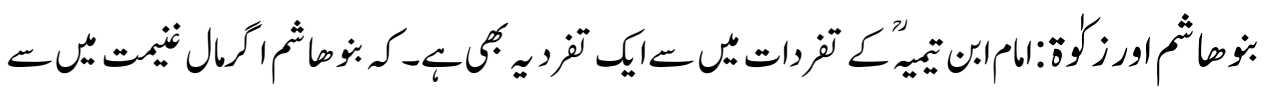

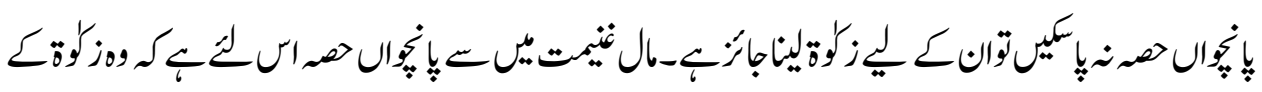

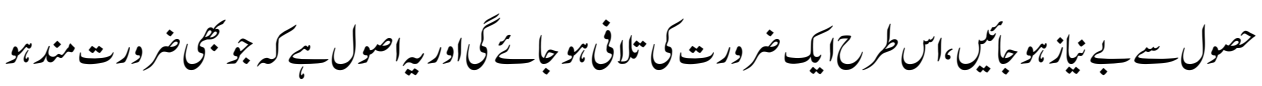

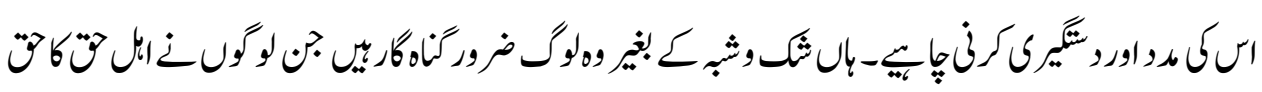

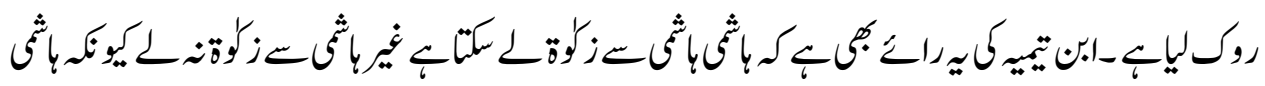

${ }^{30}$ Ba'ali, Al-Akhbar Al-Alamiya from Al-Akhbaraat Al-Qafhiyat, p: 154

${ }^{31}$ Abu Zahra, Muhammad ibn Ahmad (1898-1974) Ibn Taymiyyah Hayate-Asra, Arawah wa Fuqah Dar al-Fikr al-Arabi, Al-Cairo, Egypt p_342 


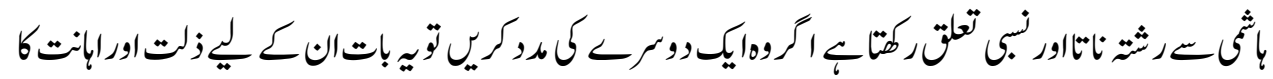

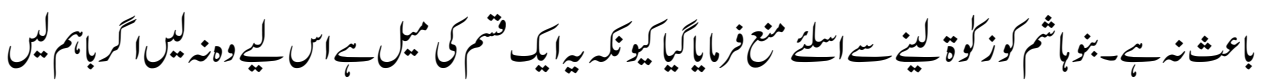

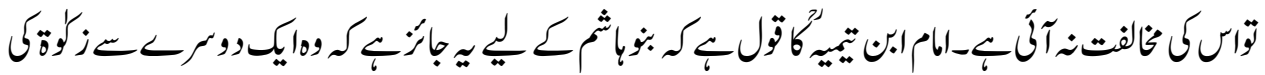

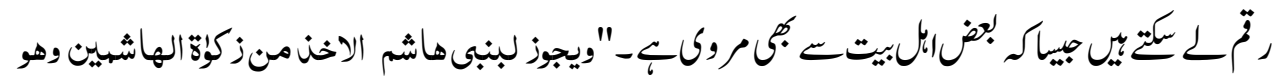

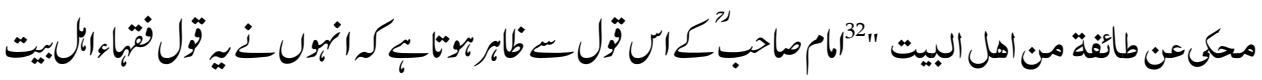

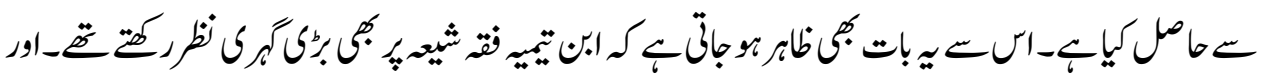

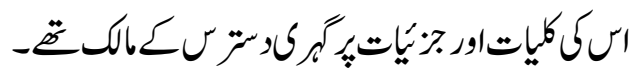

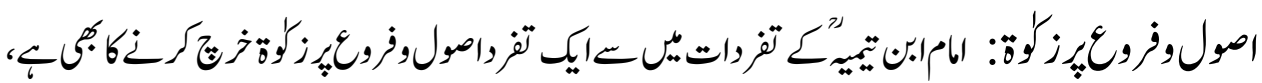

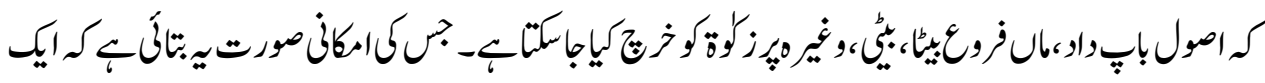

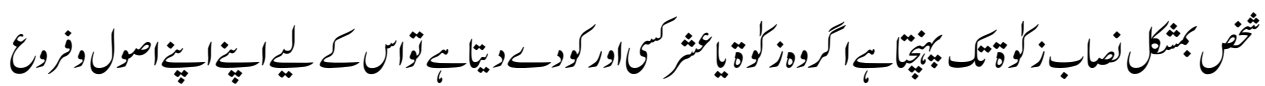

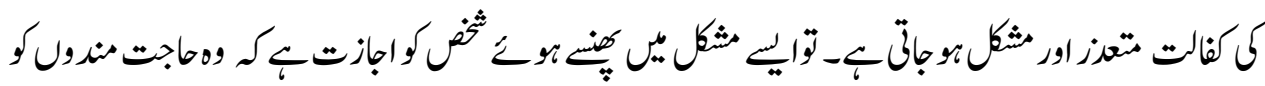

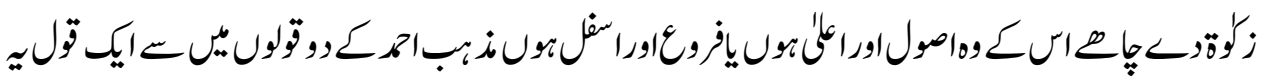

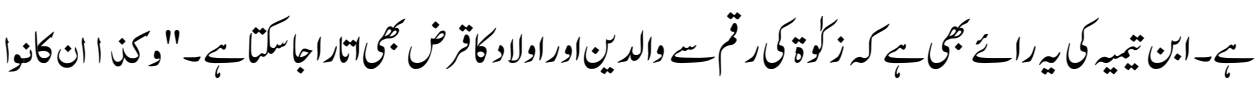

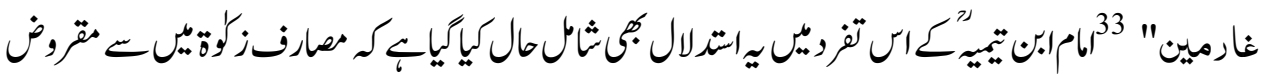

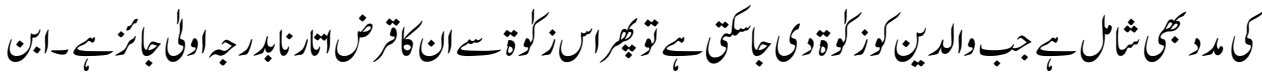

${ }^{32}$ Ba'ali, Aladdin Abu al-Hasan Ali ibn Muhammad ibn Abbas Damascus Hanbali (d. 803 AH) Al-Asimah, p. 154.

33 Ibn Taymiyyah, Taqi al-Din Ahmad ibn Abdul Halim, Collection of Fatwas of Ibn Taymiyyah, Dar al-Kitab al-'Ulamiyah, Beirut, Lebanon, 1403 AH-1983 AD, fourth volume, second section, 4/61. 


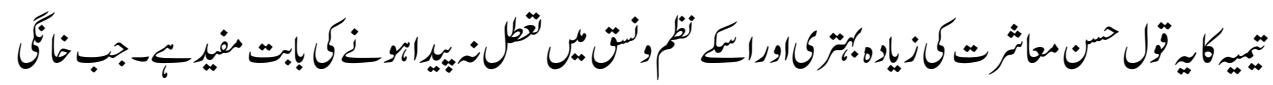

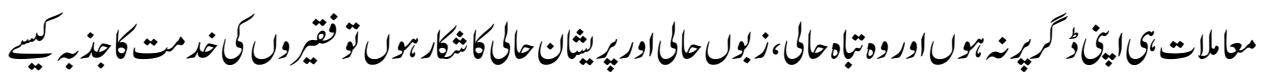

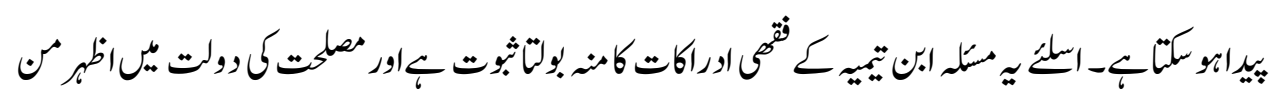

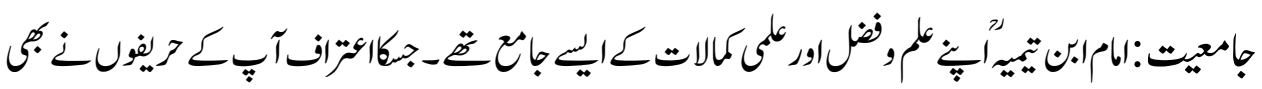

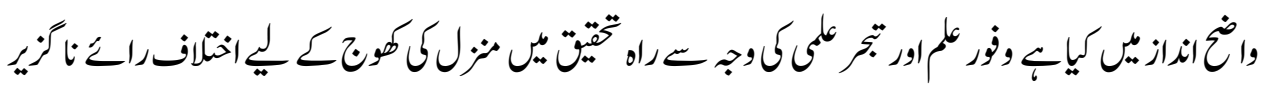

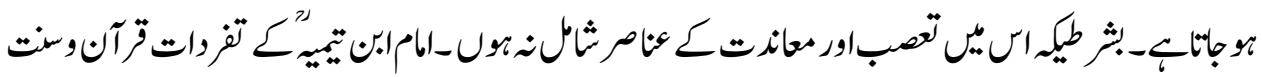

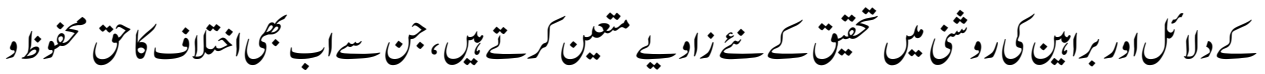

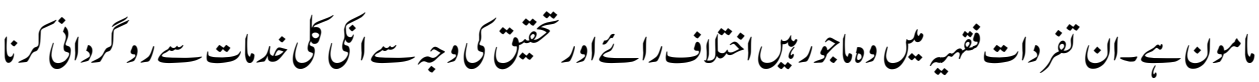

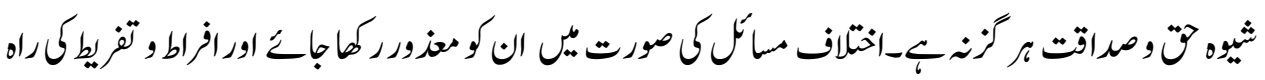

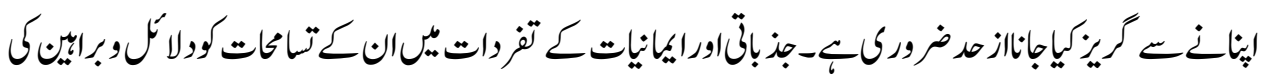

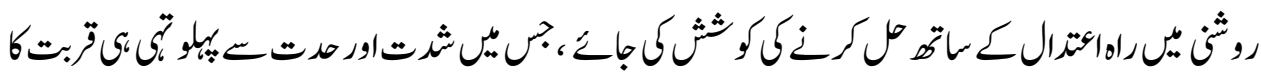

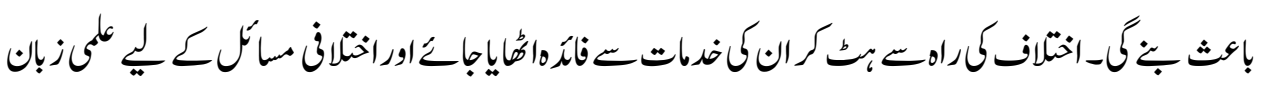

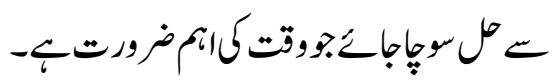

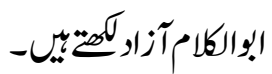

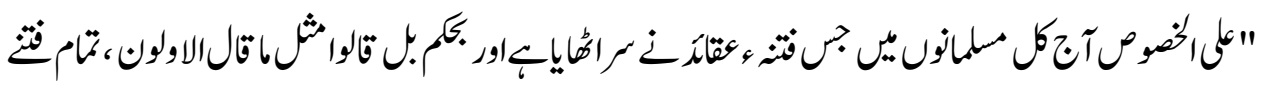

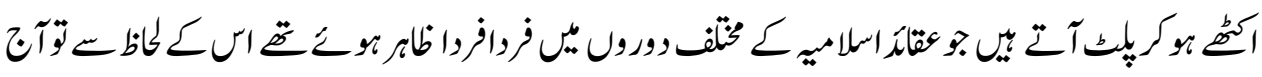




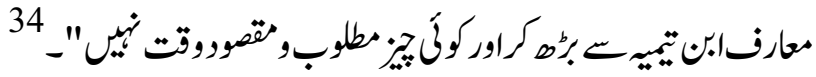

$$
\begin{aligned}
& \text { خلاص .كث: }
\end{aligned}
$$

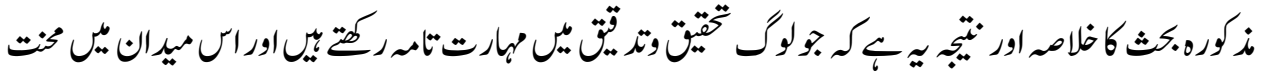

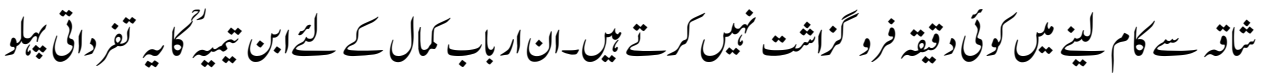

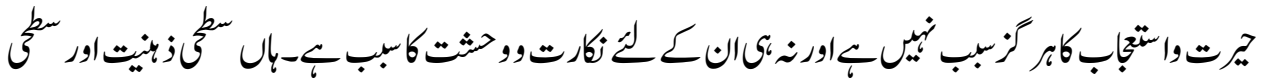

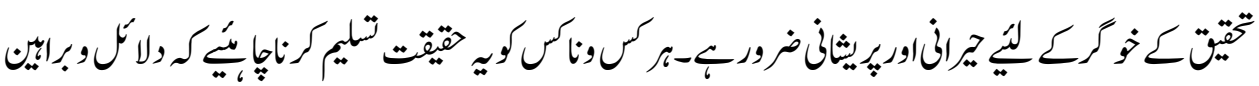

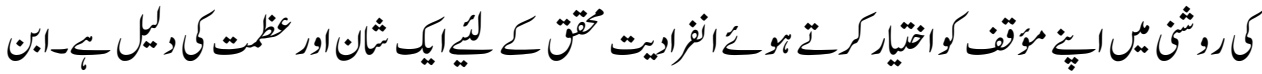

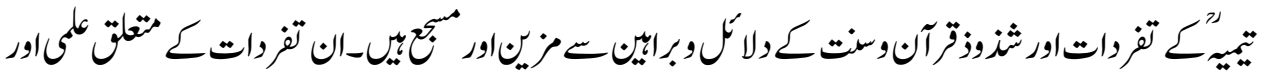

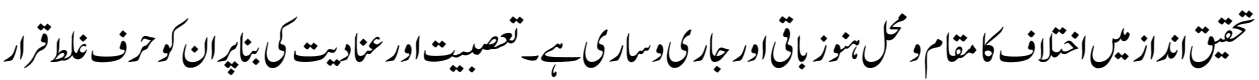

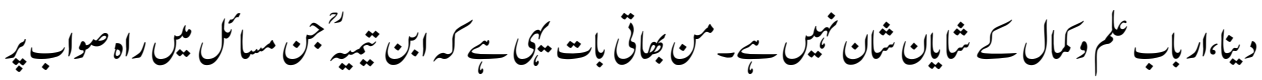

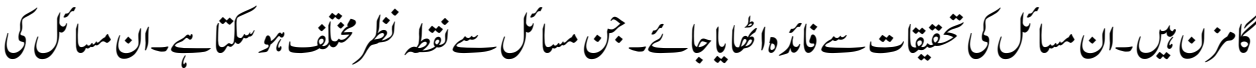

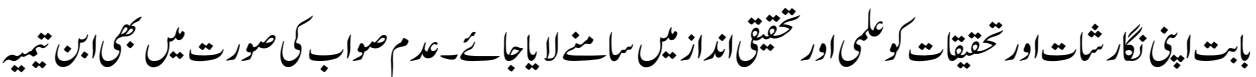

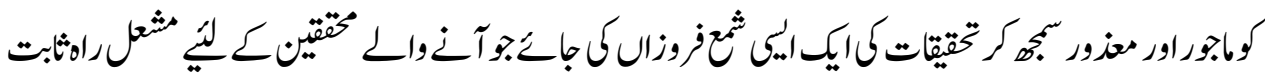

\section{(9)(1)(9)}

Attribution-NonCommercial-ShareAlike 4.0 International (CC BY-NC-SA 4.0)

34 Azad, Abu Al-Kalam, Tazkira, Compiled by: Malik Ram, Maktab-eJamal, Urdu Bazaar, Lahore, Pakistan. 2014 Pp. 179 to 180. 